\title{
Hybrid B-Spline Collocation Method for Solving the Generalized Burgers-Fisher and Burgers-Huxley Equations
}

\author{
Imtiaz Wasim, ${ }^{1}$ Muhammad Abbas $\mathbb{D D}^{1},{ }^{1}$ and Muhammad Amin ${ }^{2}$ \\ ${ }^{1}$ Department of Mathematics, University of Sargodha, Sargodha 40100, Pakistan \\ ${ }^{2}$ Department of Mathematics, National College of Business Administration and Economics, Lahore 54000, Pakistan \\ Correspondence should be addressed to Muhammad Abbas; m.abbas@uos.edu.pk
}

Received 17 August 2017; Revised 5 November 2017; Accepted 13 December 2017; Published 18 January 2018

Academic Editor: Chaudry M. Khalique

Copyright (c) 2018 Imtiaz Wasim et al. This is an open access article distributed under the Creative Commons Attribution License, which permits unrestricted use, distribution, and reproduction in any medium, provided the original work is properly cited.

\begin{abstract}
In this study, we introduce a new numerical technique for solving nonlinear generalized Burgers-Fisher and Burgers-Huxley equations using hybrid B-spline collocation method. This technique is based on usual finite difference scheme and Crank-Nicolson method which are used to discretize the time derivative and spatial derivatives, respectively. Furthermore, hybrid B-spline function is utilized as interpolating functions in spatial dimension. The scheme is verified unconditionally stable using the Von Neumann (Fourier) method. Several test problems are considered to check the accuracy of the proposed scheme. The numerical results are in good agreement with known exact solutions and the existing schemes in literature.
\end{abstract}

\section{Introduction}

Nonlinear partial differential equations (PDEs) play a significant role in different fields of science and engineering. Many physical problems are designed in mathematical form using nonlinear PDEs [1-3]. The generalized Burgers-Fisher (GBF) and generalized Burgers-Huxley (GBH) equations describe many physical phenomena. Numerical treatment of these two equations have become a dominant tool due to complexities in finding their solutions.

Consider one-dimensional nonlinear partial differential equation of the following form:

$$
u_{t}+\alpha u^{\delta} u_{x}-\epsilon u_{x x}=\beta f(u), \quad a \leq x \leq b, 0 \leq t \leq T
$$

subject to the initial condition,

$$
u(x, 0)=g(x), \quad a \leq x \leq b,
$$

and the boundary conditions,

$$
\begin{aligned}
& u(a, t)=g_{1}(t), \\
& u(b, t)=g_{2}(t),
\end{aligned}
$$

$$
0 \leq t \leq T
$$

where $u=u(x, t)$ and $f(u)$ are some nonlinear expressions in terms of $u(x, t)$ provided that $f(u)=u\left(1-u^{\delta}\right)$ for GBF equation and $f(u)=u\left(1-u^{\delta}\right)\left(u^{\delta}-\gamma\right)$ for GBH equation while $\alpha, \beta, \gamma, \delta, \epsilon$ are constants such that $\alpha, \beta \geq 0, \delta>0, \gamma \in(0,1)$, $\epsilon \in(0,1]$. The nonlinear diffusion models generated from (1) have a significant role in nonlinear physics and of great practical interest.

1.1. Model I. The GBF equation has a lot of applications in the fields such as fluid mechanics [4], gas dynamics, plasma physics [4], number theory, elasticity [5], and heat conduction [6]. This equation becomes the classical Fisher equation when $\alpha=0, \delta=1$ which is one of the significant structures in population biology and is given by

$$
u_{t}-\epsilon u_{x x}=\beta u(1-u) .
$$

Kolmogorov et al. [7] wrote down the same equation for the description of dynamic spread of a combustion front. It arises in several phenomena involving perturbation spreads in excitable mediums, spreading of bacterial colonies [8], spread of reaction fronts in chemically bistable systems [9], and switching in nonlinear optics [10]. 
The exact solution of GBF is

$$
u(x, t)=\left(\frac{\gamma}{2}+\frac{\gamma}{2} \tanh \left(d_{1}\left(x-d_{2} t\right)\right)\right)^{1 / \delta},
$$

subject to the initial condition,

$$
u(x, 0)=\left(\frac{\gamma}{2}+\frac{\gamma}{2} \tanh \left(d_{1} x\right)\right)^{1 / \delta},
$$

and the boundary conditions,

$$
\begin{aligned}
& u(a, t)=\left(\frac{\gamma}{2}+\frac{\gamma}{2} \tanh \left(d_{1}\left(a-d_{2} t\right)\right)\right)^{1 / \delta} \\
& u(b, t)=\left(\frac{\gamma}{2}+\frac{\gamma}{2} \tanh \left(d_{1}\left(b-d_{2} t\right)\right)\right)^{1 / \delta},
\end{aligned}
$$

where

$$
\begin{aligned}
& d_{1}=\frac{-\alpha+\sqrt{\alpha^{2}+4 \beta(1+\delta)}}{4(1+\delta)} \gamma \delta, \\
& d_{2}=\frac{\gamma \alpha}{(1+\delta)}-\frac{(1+\delta-\gamma)\left(-\alpha+\sqrt{\alpha^{2}+4 \beta(1+\delta)}\right)}{2(1+\delta)} .
\end{aligned}
$$

Wang et al. [11] investigated the exact solution of $\mathrm{GBH}$ equation with the help of nonlinear transformations.

1.2. Model II. Satsuma et al. [12] investigated the GBH equation in 1987. This equation reduces to the Huxley equation [11] when $\alpha=0, \delta=1, \epsilon=1$ which describes nerve pulse propagation in nerve fibres and wall motion in liquid crystals $[13,14]$. It can be expressed as follows:

$$
u_{t}-u_{x x}=\beta u(1-u)(u-\gamma) .
$$

By considering a well-known experiment in liquid crystals, a similarity between the motion of a wall in liquid crystals and nerve propagation was discussed in [14]. These models have been studied widely in the last decades due to their importance in neurobiology. Hodgkin and Huxley [15] suggested their famous Hodgkin-Huxley model for nerve propagation in 1952. It takes the form of Burgers equation by considering $\beta=0, \delta=1, \epsilon=1$. In nonlinear dissipative systems [16], it describes the far field of wave propagation and can be expressed as follows:

$$
u_{t}+\alpha u u_{x}-u_{x x}=0 .
$$

It becomes a FitzHugh-Nagumo (FN) equation when $\alpha=0$, $\beta=1, \delta=1, \epsilon=1$ are chosen. Basically, it is reaction diffusion equation utilized in circuit theory and biology [17] and its mathematical form is

$$
u_{t}-u_{x x}=u(1-u)(u-\gamma) .
$$

When $\alpha \neq 0, \beta \neq 0, \delta=1$, this equation also reduces to prototype model named as Burgers-Huxley equation. It describes the interaction between diffusion transports, convection, and reaction mechanisms [18] and is given by

$$
u_{t}+\alpha u u_{x}-\epsilon u_{x x}=\beta u(1-u)(u-\gamma) .
$$

The exact solution of GBH equation can be written as follows:

$$
u(x, t)=\left(0.5+0.5 \tanh \left(\sigma_{1}\left(x-\sigma_{2} t\right)\right)\right)^{1 / \delta},
$$

subject to the initial condition,

$$
u(x, 0)=\left(0.5+0.5 \tanh \left(\sigma_{1} x\right)\right)^{1 / \delta},
$$

and the boundary conditions,

$$
\begin{aligned}
& u(a, t)=\left(0.5+0.5 \tanh \left(\sigma_{1}\left(a-\sigma_{2} t\right)\right)\right)^{1 / \delta}, \\
& u(b, t)=\left(0.5+0.5 \tanh \left(\sigma_{1}\left(b-\sigma_{2} t\right)\right)\right)^{1 / \delta},
\end{aligned}
$$

where

$$
\begin{aligned}
& \sigma_{1}=\frac{-\alpha \delta}{2(1+\delta)} \\
& \sigma_{2}=\frac{\alpha}{(1+\delta)}+\frac{\beta(1+\delta)}{\alpha}
\end{aligned}
$$

This solution was investigated by Xinyi and Yuekai [19] which is the generalization of the preceding results.

Several numerical techniques have been developed to find the numerical solution of GBF and GBH equations. Javidi presented the numerical solution of $\mathrm{GBH}$ equation using spectral collocation method [20] and pseudospectral and preconditioning [21] and Chebyshev polynomials to develop a new domain decomposition algorithm [22]. Golbabai and Javidi [23] applied a spectral domain decomposition technique for the numerical solution of GBF equation. Darvishi et al. [24] investigated the numerical solution of GBH equation by adopting a spectral collocation method and Darvishi et al.s preconditioning. Sari et al. [25] presented the numerical solution of GBF equation by applying a compact finite difference scheme. Hammad and El-Azab [26] computed the numerical solution of two types of equations, namely, GBF and $\mathrm{GBH}$, using $2 \mathrm{~N}$ order compact finite difference scheme. A computational meshless method was developed by Khattak [27] for solving the GBH equation.

Sari and Gurarslan [28] obtained the numerical solution of the $\mathrm{GBH}$ equation using a polynomial differential quadrature method. Malik et al. [29] developed a heuristic scheme for the numerical solution of the GBF equation based on the hybridization of Exp-function method with nature inspired algorithm. The problem was converted into a nonlinear ordinary differential equation (ODE) by substitution. The travelling wave solution was approximated by the Expfunction method with unknown parameters. Dehghan et al. [30] developed two numerical methods based on the interpolating scaling functions and mixed collocation finite difference schemes for the numerical solution of the $\mathrm{GBH}$ equation.

Zhang et al. [31] developed a new kind of exact finite difference scheme for solving Burgers equation and BurgersFisher equation using the solitary wave solution. Biazar and Muhammadi [32] solved GBH equation using differential transform method (DTM). Bratsos [33, 34] solved GBH equation using a modified predictor-corrector method based 
on a second- and fourth-order time finite difference scheme. Zarebnia and Aliniya [35] used a mesh-free collocation method based on sinc functions for solving the BurgersHuxley equation. Batiha et al. [36] applied He's variational iteration method (VIM) without any discretization to solve the GBH equation. Morufu [37] developed an improved algorithm for solving GBF equation based on a Maple code. Hashim et al. [38] applied Adomian decomposition method (ADM) to get rapidly convergent analytical series solution of the GBH equation.

Zhao et al. [39] approximated the GBF equation using the pseudospectral method based on Crank-Nicolson/leapfrog scheme. The approximate solutions were obtained for the $\mathrm{GBH}$ and GBF equations using the Adomian and discrete Adomian decomposition methods [40, 41]. Inan and Bahadir [42] obtained a numerical solution of the $\mathrm{GBH}$ equation using implicit exponential finite difference method. Celik [43] proposed a Chebyshev wavelet collocation method based on truncated Chebyshev wavelet series for the solution of $\mathrm{GBH}$ equation. Moreover, the numerical solution of proposed GBH equation was obtained using several numerical methods named Galerkin method [44], implicit and fully implicit exponential finite difference methods [45], Haar wavelet method [46], conditionally bounded and symmetrypreserving method [47], linearly implicit compact scheme [48], positive and bounded finite element method [49], explicit solution scheme [50], exponential time differencing scheme [51], and higher order finite difference schemes [52].

The B-spline collocation scheme is a well-known interpolating or approximating scheme which provides a good approximation rate, computationally fast, numerically consistent, and has ability to reproduce the shape of the data with second order of continuity as compared to polynomials. Recently, several numerical schemes based on different types of B-spline functions were applied to find the numerical solutions of the differential equations. Mittal and Tripathi [53] proposed a numerical scheme based on modified cubic B-spline functions to get the approximate solutions of GBF and GBH equations. Mittal and Jain [54] obtained a numerical solution of nonlinear Burgers equation using a modified cubic B-spline collocation method. Singh et al. [55] developed a numerical scheme for solving the $\mathrm{GBH}$ equation using modified cubic B-spline differential quadrature method (MCB-DQM) and numerical results can be obtained using SSP-RK43 scheme. Reza [56] implemented the cubic B-spline collocation scheme based on the finite difference scheme for solving the GBH equation. Reza [57] developed a numerical method based on exponential B-spline with finite difference approximations to solve the GBF equation. Recently, Bukhari et al. [58] applied local radial basis functions differential collocation (LRBDQ) method to compute the numerical solution of $\mathrm{GBH}$ equation.

1.3. Motivation of the Study. The finite difference scheme is not the only tool for computing approximations to the solution of boundary value problems. There are various approximation techniques which have been examined by many researchers. Spline interpolation method is one of the most effective approximation methods on account of its

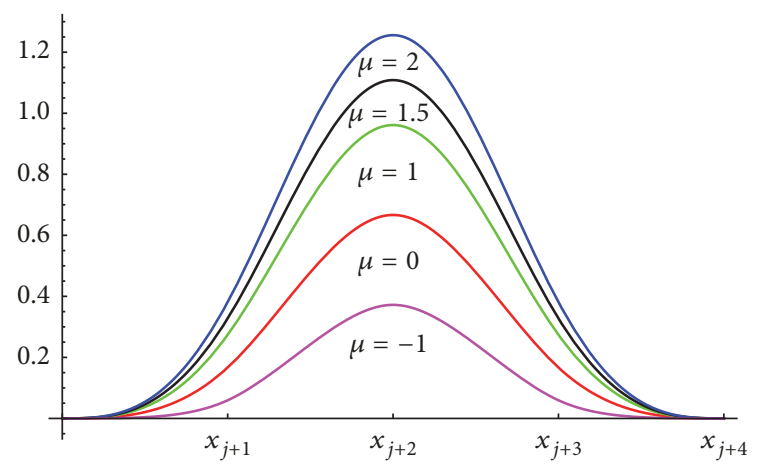

FIGURE 1: Hybrid B-spline functions with parameter $\mu=2,1.5,1,0$ and -1 .

simplicity and practicality. The main advantage of using this method is that it is able to approximate the analytical curve up to certain smoothness. Therefore, the spline method has the flexibility to get the approximation at any point in the domain with more accurate results compared to the usual finite difference method. This, thus, provides the motivation for this study on examining the accuracy of hybrid B-spline on solving nonlinear partial differential equation. However, one of the limitations of classical B-spline interpolation is that it does not possess any free parameter for the curve modification. Therefore, the shape of the curve is incapable of being altered once the control points are determined. On the other hand, spline interpolation is a global interpolation; any changes of the data point will require solving all the linear systems again. The advantage of using hybrid B-spline is that it possesses a free parameter $\mu$ to control the global shape of curve. An appropriate choice of the parameter rises the order of accuracy of the scheme. Hybrid B-spline basis function reduces to cubic trigonometric $B$-spline and cubic $B$-spline function when $\mu=0$ and 1 , respectively. This research focuses on the value of $\mu>1$. Figure 1 depicts the graph of cubic trigonometric B-spline when $\mu=0$, cubic B-spline function when $\mu=1$, and the effect of parameter $\mu$ for proposed hybrid B-spline function. Therefore, the superiority of this spline interpolation method on proposed problem is to be examined.

Although a finite difference scheme is only able to give the approximations at selected points, this method is relatively simple and very much easy to implement. Hence, an idea of combining finite difference approach with hybrid B-spline interpolation method for solving proposed problem also naturally arose. Here, hybrid B-spline is used to model the solution curve at each level of time. Thus, it is applied to interpolate the solutions at time $t$ while finite difference scheme is used to discretize the time derivative. The obtained results are more accurate than some available methods in the literature. Stability analysis of the proposed method is presented and shown to be unconditionally stable without any restriction on the choice of step sizes $h$ and $\Delta t$. An advantage of the proposed hybrid B-spline collocation method (HBSCM) outlined in this study is that it produces a spline function on each new time line which can be used to obtain the solution at any intermediate point in the spatial direction 
whereas the finite difference approach yields the solution only at the selected points.

This article is organized as follows: In Section 2, hybrid B-spline collocation method, a combination of cubic Bspline function and cubic trigonometric B-spline function with one free parameter $\mu$, is constructed and applied to obtain the numerical solutions of the proposed equations. In Section 3, the method is proved unconditionally stable by Von Neumann approach. In Section 4, several numerical cases of GBF and GBH equations are considered to show the feasibility of the proposed method. Finally, the conclusion of this study is provided.

\section{Materials and Methods}

This section introduces the hybrid B-spline basis function and derivation of proposed HBSCM for solving the GBF and GBH equations.

2.1. Hybrid B-Spline Basis Function. For the discretization of the grid region $[a, b] \times[0, T]$ an equally divided mesh $\Omega$ with grid points $\left(x_{j}, t_{k}\right)$ is considered. Here $x_{j}=a+j h, t_{k}=k \Delta t$ where $j=0,1, \ldots, n$ and $k=0,1, \ldots, N$ while $h$ and $\Delta t$ are spatial size and time step, respectively. Hybrid B-spline collocation basis function can be written as follows:

$$
\begin{aligned}
& H_{j}^{4}(x) \\
& =\left\{\begin{array}{lr}
\frac{\mu}{6 h^{3}}\left(x-x_{j}\right)^{3}+\frac{(1-\mu)}{\zeta} r^{3}\left(x_{j}\right) & x \in\left[x_{j}, x_{j+1}\right] \\
\frac{\mu}{6 h^{3}}\left(h^{3}+3 h^{2}\left(x-x_{j+1}\right)+3 h\left(x-x_{j+1}\right)^{2}-3\left(x-x_{j+1}\right)^{3}\right)+\frac{(1-\mu)}{\zeta}\left(r\left(x_{j}\right)\left(r\left(x_{j}\right) s\left(x_{j+2}\right)+s\left(x_{j+3}\right) r\left(x_{j+1}\right)\right)+s\left(x_{j+4}\right) r^{2}\left(x_{j+1}\right)\right) & x \in\left[x_{j+1}, x_{j+2}\right] \\
\frac{\mu}{6 h^{3}}\left(h^{3}+3 h^{2}\left(x_{j+3}-x\right)+3 h\left(x_{j+3}-x\right)^{2}-3\left(x_{j+3}-x\right)^{3}\right)+\frac{(1-\mu)}{\zeta}\left(s\left(x_{j+4}\right)\left(r\left(x_{j+1}\right) s\left(x_{j+3}\right)+s\left(x_{j+4}\right) r\left(x_{j+2}\right)\right)+r\left(x_{j}\right) s^{2}\left(x_{j+3}\right)\right) & x \in\left[x_{j+2}, x_{j+3}\right] \\
\frac{\mu}{6 h^{3}}\left(x_{j+4}-x\right)^{3}+\frac{(1-\mu)}{\zeta} s^{3}\left(x_{j+4}\right) & x \in\left[x_{j+3}, x_{j+4}\right] \\
0 & \text { otherwise, }
\end{array}\right.
\end{aligned}
$$

where $r\left(x_{j}\right)=\sin \left(\left(x-x_{j}\right) / 2\right), s\left(x_{j}\right)=\sin \left(\left(x_{j}-x\right) / 2\right), \zeta=$ $\sin (h / 2) \sin (h) \sin (3 h / 2)$ and $\mu \in R$.

The approximate solution $u\left(x_{j}, t_{k}\right)$ to the exact solution $u_{\text {exc }}(x, t)$ can be expressed as follows [59-64]:

$$
u_{j}^{k}(x, t)=\sum_{m=j-1}^{j+1} D_{m}^{k}(t) H_{m}^{4}(x),
$$

where $D_{m}^{k}(t)$ are time-dependent unknowns to be determined.

The values of $H_{j}^{4}(x)$ and its derivatives at node $x=x_{j}$ are given by

$$
\begin{aligned}
& H_{m}^{4}\left(x_{j}\right)= \begin{cases}a_{1}=\frac{\mu}{6}+(1-\mu) \sin ^{2}\left(\frac{h}{2}\right) \operatorname{cosec}(h) \operatorname{cosec}\left(\frac{3 h}{2}\right) & m=j \pm 1 \\
a_{2}=\frac{2 \mu}{3}+(1-\mu) \frac{2}{1+2 \cos (h)} & m=j \\
0 & \text { otherwise, }\end{cases} \\
& \frac{d}{d x} H_{m}^{4}\left(x_{j}\right)= \begin{cases}a_{3}=-\left(\frac{\mu}{2 h}+(1-\mu) \frac{3}{4} \operatorname{cosec}\left(\frac{3 h}{2}\right)\right) & m=j-1 \\
a_{4}=\left(\frac{\mu}{2 h}+(1-\mu) \frac{3}{4} \operatorname{cosec}\left(\frac{3 h}{2}\right)\right) & m=j+1 \\
0 & \text { otherwise }\end{cases} \\
& \frac{d^{2}}{d x^{2}} H_{m}^{4}\left(x_{j}\right)= \begin{cases}a_{5}=\frac{\mu}{h^{2}}+(1-\mu) \frac{3(1+3 \cos (h)) \operatorname{cosec}^{2}(h / 2)}{16(2 \cos (h / 2)+\cos (3 h / 2))} & m=j \pm 1 \\
a_{6}=-\left(\frac{2 \mu}{h^{2}}+(1-\mu) \frac{3 \cos ^{2}(h / 2) \operatorname{cosec}^{2}(h / 2)}{(2+4 \cos (h))}\right) & m=j \\
0 & \text { otherwise. }\end{cases}
\end{aligned}
$$


From (17)-(19), the values of $u_{j}^{k}(x, t)$ and their derivatives at the knots are calculated in terms of time parameters $D_{j}^{k}(t)$ as follows:

$$
\begin{aligned}
u_{j}^{k} & =a_{1} D_{j-1}^{k}+a_{2} D_{j}^{k}+a_{1} D_{j+1}^{k}, \\
\left(u_{x}\right)_{j}^{k} & =a_{3} D_{j-1}^{k}+0 D_{j}^{k}+a_{4} D_{j+1}^{k}, \\
\left(u_{x x}\right)_{j}^{k} & =a_{5} D_{j-1}^{k}+a_{6} D_{j}^{k}+a_{5} D_{j+1}^{k} .
\end{aligned}
$$

Equation (18) and boundary conditions given in (3) are used to obtain the approximate solution at end points of the mesh as

$$
\begin{aligned}
& u\left(x_{0}, t_{k+1}\right)=a_{1} D_{-1}^{k+1}+a_{2} D_{0}^{k+1}+a_{1} D_{1}^{k+1}=g_{1}\left(t_{k+1}\right), \\
& u\left(x_{n}, t_{k+1}\right)=a_{1} D_{n-1}^{k+1}+a_{2} D_{n}^{k+1}+a_{1} D_{n+1}^{k+1}=g_{2}\left(t_{k+1}\right) .
\end{aligned}
$$

2.2. Numerical Solution of the Generalized Burgers-Fisher and Generalized Burgers-Huxley Equations. By utilizing temporal discretization and Crank-Nicolson approach, (1) can be written as

$$
\begin{gathered}
\frac{u_{j}^{k+1}-u_{j}^{k}}{\Delta t}+\frac{\left(\alpha\left(u^{\delta} u_{x}\right)_{j}^{k+1}-\epsilon\left(u_{x x}\right)_{j}^{k+1}\right)}{2} \\
\quad+\frac{\left(\alpha\left(u^{\delta} u_{x}\right)_{j}^{k}-\epsilon\left(u_{x x}\right)_{j}^{k}\right)}{2} \\
=\beta\left(\frac{f\left(u_{j}^{k}\right)+f\left(u_{j}^{k+1}\right)}{2}\right),
\end{gathered}
$$

where $k$ and $k+1$ describe successive time positions and $f\left(u_{j}^{k}\right)=u_{j}^{k}\left(1-\left(u^{\delta}\right)_{j}^{k}\right)$ for GBF and $f\left(u_{j}^{k}\right)=u_{j}^{k}(1-$ $\left.\left(u_{j}^{k}\right)^{\delta}\right)\left(\left(u_{j}^{k}\right)^{\delta}-\gamma\right)$ for GBH equations. After simplification, (22) for GBF can be written as follows:

$$
\begin{aligned}
u_{j}^{k+1} & +0.5 \alpha \Delta t\left(u^{\delta} u_{x}\right)_{j}^{k+1}-0.5 \epsilon \Delta t\left(u_{x x}\right)_{j}^{k+1} \\
& -0.5 \Delta t \beta u_{j}^{k+1}\left(1-\left(u^{\delta}\right)_{j}^{k+1}\right) \\
= & u_{j}^{k}-0.5 \alpha \Delta t\left(u^{\delta} u_{x}\right)_{j}^{k}+0.5 \epsilon \Delta t\left(u_{x x}\right)_{j}^{k} \\
& +0.5 \Delta t \beta u_{j}^{k}\left(1-\left(u^{\delta}\right)_{j}^{k}\right)
\end{aligned}
$$

and (22) for $\mathrm{GBH}$ equation can be written as follows:

$$
\begin{aligned}
u_{j}^{k+1}+ & 0.5 \alpha \Delta t\left(u^{\delta} u_{x}\right)_{j}^{k+1}-0.5 \epsilon \Delta t\left(u_{x x}\right)_{j}^{k+1} \\
& -0.5 \Delta t \beta u_{j}^{k+1}\left(1-\left(u^{\delta}\right)_{j}^{k+1}\right)\left(\left(u_{j}^{k+1}\right)^{\delta}-\gamma\right) \\
= & u_{j}^{k}-0.5 \alpha \Delta t\left(u^{\delta} u_{x}\right)_{j}^{k}+0.5 \epsilon \Delta t\left(u_{x x}\right)_{j}^{k} \\
& +\Delta t \beta u_{j}^{k}\left(1-\left(u^{\delta}\right)_{j}^{k}\right)\left(\left(u_{j}^{k}\right)^{\delta}-\gamma\right) .
\end{aligned}
$$

The system, thus obtained, on simplifying (23) for GBF and (24) for $\mathrm{GBH}$ problem using (20) consists of $n+1$ nonlinear equations in $n+3$ unknowns $D^{k+1}=\left(D_{-1}^{k+1}\right.$, $\left.D_{0}^{k+1}, D_{1}^{k+1}, \ldots, D_{n+1}^{k+1}\right)$ at the time level $t_{k+1}$. Further two equations are included in the resulting system to obtain a unique solution of the problem using the boundary conditions given in (21). The initial vector $D^{0}$ can be obtained from initial condition given in (2) [59-64]. Thus, the resulting system becomes a matrix system of dimension $(n+3) \times(n+3)$ which is a tridiagonal system that can be solved by Thomas algorithm [65-67].

\section{Stability}

This section discusses the Von Neumann criteria to investigate the stability of GBF and GBH equations. In the product term, consider $u^{\delta}=\tau$ where $\tau$ is taken [68] as locally constant, the GBF equation is described as follows:

$$
u_{t}+\alpha \tau u_{x}-\epsilon u_{x x}=(1-\tau) \beta u
$$

and applying same procedure for nonlinear term as in [64], the GBH equation can be converted to the following:

$$
u_{t}+\alpha \tau u_{x}-\epsilon u_{x x}=-\gamma \beta u \text {. }
$$

Utilizing same procedure as stated in (22) and setting $1-\tau=$ $\lambda$, the above two GBF and GBH equations take the following forms:

$$
\begin{gathered}
b_{1} u_{j}^{k+1}+b_{2}\left(u_{x}\right)_{j}^{k+1}-b_{3}\left(u_{x x}\right)_{j}^{k+1} \\
=b_{4} u_{j}^{k}-b_{2}\left(u_{x}\right)_{j}^{k}+b_{3}\left(u_{x x}\right)_{j}^{k}, \\
a_{11} u_{j}^{k+1}+b_{2}\left(u_{x}\right)_{j}^{k+1}-b_{3}\left(u_{x x}\right)_{j}^{k+1} \\
=a_{22} u_{j}^{k}-b_{2}\left(u_{x}\right)_{j}^{k}+b_{3}\left(u_{x x}\right)_{j}^{k},
\end{gathered}
$$

where $a_{11}=2+\beta \gamma \Delta t, a_{22}=2-\beta \gamma \Delta t, b_{1}=2-\beta \lambda \Delta t, b_{2}=$ $\alpha \tau \Delta t, b_{3}=\epsilon \Delta t, b_{4}=2+\beta \lambda \Delta t$

Substitute (23) into (27) and simplifying yield

$$
\begin{gathered}
q_{1} D_{j-1}^{k+1}+q_{2} D_{j}^{k+1}+q_{3} D_{j+1}^{k+1} \\
=q_{4} D_{j-1}^{k}+q_{5} D_{j}^{k}+q_{6} D_{j+1}^{k}, \\
p_{1} D_{j-1}^{k+1}+p_{2} D_{j}^{k+1}+p_{3} D_{j+1}^{k+1} \\
=p_{4} D_{j-1}^{k}+p_{5} D_{j}^{k}+p_{6} D_{j+1}^{k},
\end{gathered}
$$

where

$$
\begin{aligned}
& p_{1}=a_{11} a_{1}+b_{2} a_{3}-b_{3} a_{5}, \\
& p_{2}=a_{11} a_{2}-b_{3} a_{6}, \\
& p_{3}=a_{11} a_{1}+b_{2} a_{4}-b_{3} a_{5}, \\
& p_{4}=a_{22} a_{1}-b_{2} a_{3}+b_{3} a_{5}, \\
& p_{5}=a_{22} a_{2}+b_{3} a_{6},
\end{aligned}
$$




$$
\begin{aligned}
& p_{6}=a_{22} a_{1}-b_{2} a_{4}+b_{3} a_{5}, \\
& q_{1}=b_{1} a_{1}+b_{2} a_{3}-b_{3} a_{5}, \\
& q_{2}=b_{1} a_{2}-b_{3} a_{6}, \\
& q_{3}=b_{1} a_{1}+b_{2} a_{4}-b_{3} a_{5}, \\
& q_{4}=b_{4} a_{1}-b_{2} a_{3}+b_{3} a_{5}, \\
& q_{5}=b_{4} a_{2}+b_{3} a_{6}, \\
& q_{6}=b_{4} a_{1}-b_{2} a_{4}+b_{3} a_{5} .
\end{aligned}
$$

Now substituting $D_{j}^{k}=\rho^{k} e^{i \omega j h}$ into (28). After simplification dividing both sides by $\rho^{k} e^{i \omega j h}$, we obtain the following expressions given in (29) and (30) for GBF and GBH equations, respectively:

$$
\begin{aligned}
& \rho=\frac{c_{1}+i d_{1}}{c_{2}+i d_{2}}, \quad i=\sqrt{-1}, \\
& \rho=\frac{l_{1}+i m_{1}}{l_{2}+i m_{2}}, \quad i=\sqrt{-1},
\end{aligned}
$$

where

$$
\begin{aligned}
& c_{1}=\cos (\phi)\left(q_{6}+q_{4}\right)+q_{5}, \\
& d_{1}=\sin (\phi)\left(q_{6}-q_{4}\right) \\
& c_{2}=\cos (\phi)\left(q_{3}+q_{1}\right)+q_{2}, \\
& d_{2}=\sin (\phi)\left(q_{3}-q_{1}\right) \\
& l_{1}=\cos (\phi)\left(p_{6}+p_{4}\right)+p_{5}, \\
& m_{1}=\sin (\phi)\left(p_{6}-p_{4}\right) \\
& l_{2}=\cos (\phi)\left(p_{3}+p_{1}\right)+p_{2}, \\
& m_{2}=\sin (\phi)\left(p_{3}-p_{1}\right)
\end{aligned}
$$

Since the wave number $\omega=2 \pi / \lambda$ where $\lambda$ is the wave length so $\phi=\omega h=2 \pi / N$ for $0<\phi<\pi$. The amplification factor $\rho$ is a complex number; therefore the stability condition $|\rho| \leq 1$ yields the following relation by adopting same procedure as in $[60]$.

For GBF equation,

$$
|\rho|=\sqrt{\left(\frac{c_{1} c_{2}+d_{1} d_{2}}{c_{2}^{2}+d_{2}^{2}}\right)^{2}+\left(\frac{d_{1} c_{2}-c_{1} d_{2}}{c_{2}^{2}+d_{2}^{2}}\right)^{2}} \leq 1
$$

and, for GBH equation,

$$
|\rho|=\sqrt{\left(\frac{l_{1} l_{2}+m_{1} m_{2}}{l_{2}^{2}+m_{2}^{2}}\right)^{2}+\left(\frac{m_{1} l_{2}-l_{1} m_{2}}{l_{2}^{2}+m_{2}^{2}}\right)^{2}} \leq 1
$$

Substituting the values into (31) and (32), we obtain the following expressions for GBF equation:

$$
\begin{aligned}
& |\rho| \\
& =\sqrt{\frac{q_{4}^{2}+q_{5}^{2}+q_{6}^{2}+2 q_{5}\left(q_{4}+q_{6}\right) \cos (\phi)+2 q_{4} q_{6} \cos (2 \phi)}{q_{1}^{2}+q_{2}^{2}+q_{3}^{2}+2 q_{2}\left(q_{1}+q_{3}\right) \cos (\phi)+2 q_{1} q_{3} \cos (2 \phi)}}
\end{aligned}
$$

and, for $\mathrm{GBH}$ equation,

$$
\begin{aligned}
& |\rho| \\
& =\sqrt{\frac{p_{4}^{2}+p_{5}^{2}+p_{6}^{2}+2 p_{5}\left(p_{4}+p_{6}\right) \cos (\phi)+2 p_{4} p_{6} \cos (2 \phi)}{p_{1}^{2}+p_{2}^{2}+p_{3}^{2}+2 p_{2}\left(p_{1}+p_{3}\right) \cos (\phi)+2 p_{1} p_{3} \cos (2 \phi)}} .
\end{aligned}
$$

Taking extreme value $\phi=\pi$, (33) and (34) become

$$
\begin{aligned}
& |\rho|=\sqrt{\frac{\left(q_{4}-q_{5}+q_{6}\right)^{2}}{\left(q_{1}-q_{2}+q_{3}\right)^{2}}}, \\
& |\rho|=\sqrt{\frac{\left(p_{4}-p_{5}+p_{6}\right)^{2}}{\left(p_{1}-p_{2}+p_{3}\right)^{2}}} .
\end{aligned}
$$

By substituting the values from (28) into (35) amplification factor $\rho$ for GBF equation is

$$
\begin{aligned}
& |\rho| \\
& =\frac{\left(\xi_{2}+\xi_{5}+\xi_{9}\right)-\left(\xi_{1}+\xi_{3}+\xi_{4}+\xi_{6}+\xi_{7}+\xi_{8}+\xi_{10}\right)}{\left(\xi_{1}+\xi_{2}+\xi_{3}+\xi_{4}+\xi_{5}+\xi_{6}+\xi_{7}+\xi_{10}\right)-\left(\xi_{8}+\xi_{9}\right)}
\end{aligned}
$$

and for $\mathrm{GBH}$ equation

$|\rho|$

$$
=\frac{\left(\delta_{5}+\delta_{2}\right)-\left(\delta_{1}+\delta_{3}+\delta_{4}+\delta_{6}+\delta_{7}+3 h^{2}(1-\mu)(8+(3-4 \beta \gamma) \Delta t+(-8+(9+4 \beta \gamma) \Delta t) \cos (h)) \csc ^{2}(h / 2) \sec (h / 2)\right)}{\delta_{1}+\delta_{2}+\delta_{3}+\delta_{4}+\delta_{5}+\delta_{6}+\delta_{7}+6 h^{2}(1-\mu)\left((-8-(9+4 \beta \gamma) \Delta t)+6 \Delta t \csc ^{2}(h / 2)\right) \sec (h / 2)},
$$

where

$$
\begin{aligned}
& \xi_{1}=96 \Delta t \mu \epsilon, \\
& \xi_{2}=16 h^{2}(6-5 \mu), \\
& \xi_{3}=8 h^{2} \beta \Delta t(6-5 \mu) \lambda, \\
& \xi_{4}=192 \Delta t \epsilon \mu \cos (h),
\end{aligned}
$$

$$
\begin{aligned}
& \xi_{5}=32 h^{2} \mu \cos (h), \\
& \xi_{6}=16 h^{2} \beta \Delta t \mu \lambda \cos (h), \\
& \xi_{7}=36 h^{2} \epsilon \Delta t(1-\mu) \cot ^{2}(h / 2), \\
& \xi_{8}=48 h^{2}(1-\mu) \sec (h / 2), \\
& \xi_{9}=24 h^{2}(1-\mu) \beta \Delta t \lambda \sec (h / 2),
\end{aligned}
$$


TABLE 1: Absolute errors of GBH equation at different values of time and parameter $\mu$ taking $\Delta t=0.0001$ for case 1 .

\begin{tabular}{ccccc}
\hline$x$ & $t$ & $\mu=1.1$ & $\mu=1.5$ & $\mu=1.9$ \\
\hline & 0.05 & $7.722 E-09$ & $7.703 E-09$ & $7.684 E-09$ \\
0.1 & 0.1 & $1.129 E-08$ & $1.126 E-08$ & $1.123 E-08$ \\
& 1.0 & $1.685 E-08$ & $1.681 E-08$ & $1.677 E-08$ \\
& 5.0 & $1.685 E-08$ & $1.681 E-08$ & $1.677 E-08$ \\
& 10 & $1.685 E-08$ & $1.681 E-08$ & $1.677 E-08$ \\
& 0.05 & $1.734 E-08$ & $1.730 E-08$ & $1.726 E-08$ \\
0.5 & 0.1 & $2.881 E-08$ & $2.874 E-08$ & $2.867 E-08$ \\
& 1.0 & $4.682 E-08$ & $4.670 E-08$ & $4.658 E-08$ \\
& 5.0 & $4.682 E-08$ & $4.670 E-08$ & $4.658 E-08$ \\
& 10 & $4.682 E-08$ & $4.670 E-08$ & $7.658 E-08$ \\
& 0.05 & $7.722 E-09$ & $7.703 E-09$ & $1.684 E-09$ \\
0.9 & $1.129 E-08$ & $1.126 E-08$ & $1.123 E-08$ \\
& 1.0 & $1.685 E-08$ & $1.681 E-08$ & $1.677 E-08$ \\
& 5.0 & $1.686 E-08$ & $1.681 E-08$ & $1.677 E-08$ \\
\hline
\end{tabular}

$\xi_{10}=18 h^{2}(1-\mu) \epsilon \Delta t(1+3 \cos (h)) /(1-\cos (h)) \sec (h /$

2),

$\delta_{1}=96 \Delta t \mu$,

$\delta_{2}=16 h^{2}(6-5 \mu)$,

$\delta_{3}=8 h^{2} \beta \gamma \Delta t(6-5 \mu)$,

$\delta_{4}=192 \Delta t \mu \cos (h)$,

$\delta_{5}=32 h^{2} \mu \cos (h)$,

$\delta_{6}=16 h^{2} \beta \gamma \Delta t \mu \cos (h)$,

$\delta_{7}=36 h^{2} \Delta t(1-\mu) \cot ^{2}(h / 2)$.

Since the numerator is less than the denominator in (36) and (37), so amplification factor $|\rho| \leq 1$ for both GBF and $\mathrm{GBH}$ equations which clearly demonstrate that the proposed scheme is unconditionally stable.

\section{Numerical Results and Discussion}

This section presents the numerical results of GBF and GBH equations with initial (2) and boundary conditions (3) by HBSCM. To test the accuracy of present method, several numerical tests for different values of $\alpha, \beta, \gamma, \delta$ are provided where $L_{\infty}, L_{2}$, and order of convergence $p$ are calculated by

$$
\begin{aligned}
L_{\infty} & =\max \left|u_{j}-u_{\text {exc } j}\right|, \\
L_{2} & =\frac{\sqrt{\sum_{j=1}^{n}\left|u_{j}-u_{\text {exc } j}\right|^{2}}}{\sqrt{\sum_{j=1}^{n}\left|u_{\text {exc } j}\right|^{2}}} \\
p & =\frac{\log \left(L_{\infty}(n) / L_{\infty}(2 n)\right)}{\log (2)} .
\end{aligned}
$$

Numerical results obtained by HBSCM are compared with given exact solutions and the approximate methods existing in the literature. The programming of the proposed problem is carried out in Matlab R2015b. Numerical results are computed at different time levels with smaller storage which are tabulated in different Tables.

Consider the following numerical test cases for GBH and GBF equations to show the accuracy of proposed method.

\subsection{Numerical Test Cases for Model I}

(1) When $\alpha=1, \beta=1, \gamma=0.001, \epsilon=1$, GBH equation can be described as follows:

$$
u_{t}+u^{\delta} u_{x}-u_{x x}=u\left(1-u^{\delta}\right)\left(u^{\delta}-0.001\right) \text {. }
$$

The obtained errors at $\delta=1$ corresponding to different values of parameter $\mu$ are listed in Table 1 . Table 2 shows a comparison of the absolute errors at $\mu=3.9, \delta=1,2,3$ obtained by proposed method HBSCM with the methods existing in the literature named compact finite difference scheme (CFDS) [26], a fourth-order improved numerical scheme (FONS) [33], variational iteration method (VIM) [36], Adomian decomposition method (ADM) [38, 40], implicit exponential finite difference method (IEFM) [42], and modified cubic B-spline (MCBS) [55]. The obtained results are compared with Haar wavelet method (HWM) [46] at $t=0.8$ in Table 3 while a comparison between HBSCM and a new domain decomposition method (NDDA) [22] can be observed in Table 4 . The results of the proposed method in terms of errors comparative to Local Radial Basis Function Differential Collocation method (LRBFDQ) [58] is provided in Table 5. It can be observed that increase in $\Delta t$ did not disturb the accuracy of HBSCM and our method still approximates the exact solution quite adequately due to hybrid parameter. The graphical representations of error terms at different time levels have been carried out in Figures 2 and 3. A prominent difference in the 
TABLE 2: Comparison of absolute errors calculated by HBSCM with the existing methods at $\delta=1, \mu=3.9$ for case 1 .

\begin{tabular}{|c|c|c|c|c|c|c|c|c|c|}
\hline \multirow[b]{2}{*}{$x$} & \multicolumn{3}{|c|}{$t=0.05$} & \multicolumn{3}{|c|}{$t=0.1$} & \multicolumn{3}{|c|}{$t=1.0$} \\
\hline & 0.1 & 0.5 & 0.9 & 0.1 & 0.5 & 0.9 & 0.1 & 0.5 & 0.9 \\
\hline \multicolumn{10}{|c|}{$\delta=1$} \\
\hline HBSCM & $7.587 E-09$ & $1.704 E-08$ & $7.587 E-09$ & $1.109 E-08$ & $2.830 E-08$ & $1.109 E-08$ & $1.656 E-08$ & $4.599 E-08$ & $1.656 E-08$ \\
\hline CFDS [26] & $7.700 E-09$ & $1.728 E-08$ & $7.700 E-09$ & $1.126 E-08$ & $2.873 E-08$ & $1.126 E-08$ & $1.686 E-08$ & $4.684 E-08$ & $1.686 E-08$ \\
\hline FONS [33] & $1.264 E-09$ & $1.977 E-08$ & $4.602 E-08$ & $6.395 E-09$ & $3.996 E-08$ & $7.663 E-08$ & $3.292 E-07$ & $3.792 E-07$ & $4.292 E-07$ \\
\hline VIM [36] & $1.874 E-08$ & $1.874 E-08$ & $1.874 E-08$ & $3.748 E-08$ & $1.374 E-08$ & $3.748 E-08$ & $3.748 E-07$ & $3.748 E-07$ & $3.748 E-07$ \\
\hline $\mathrm{ADM}[38]$ & $1.874 E-08$ & $1.874 E-08$ & $1.874 E-08$ & $3.748 E-08$ & $3.748 E-08$ & $3.748 E-08$ & $3.748 E-07$ & $3.748 E-07$ & $3.748 E-07$ \\
\hline ADM [40] & $1.937 E-07$ & $1.937 E-07$ & $1.937 E-07$ & $3.874 E-07$ & $3.874 E-07$ & $3.874 E-07$ & $3.875 E-06$ & $3.875 E-06$ & $3.875 E-06$ \\
\hline IEFM [42] & $1.544 E-08$ & $3.469 E-08$ & $1.544 E-08$ & $2.258 E-08$ & $5.764 E-08$ & $2.259 E-08$ & $3.372 E-08$ & $9.369 E-08$ & $3.373 E-08$ \\
\hline MCBS [55] & - & - & - & $1.111 E-08$ & $2.870 E-08$ & $1.111 E-08$ & $1.668 E-08$ & $4.665 E-08$ & $1.668 E-08$ \\
\hline \multicolumn{10}{|c|}{$\delta=2$} \\
\hline HBSCM & $3.545 E-07$ & $7.960 E-07$ & $3.544 E-07$ & $5.182 E-07$ & $1.322 E-06$ & $5.182 E-07$ & $7.734 E-07$ & $2.148 E-06$ & $7.734 E-07$ \\
\hline VIM [36] & - & - & - & $5.515 E-05$ & $5.510 E-05$ & - & - & - & - \\
\hline ADM [38] & - & - & - & $5.515 E-05$ & $5.511 E-05$ & - & - & - & - \\
\hline IEFM [42] & $1.402 E-06$ & $3.150 E-06$ & $1.402 E-06$ & $2.051 E-06$ & $5.233 E-06$ & $2.051 E-06$ & $3.056 E-06$ & $8.490 E-06$ & $3.056 E-06$ \\
\hline \multicolumn{10}{|c|}{$\delta=3$} \\
\hline HBSCM & $1.294 E-06$ & $2.906 E-06$ & $1.293 E-06$ & $1.892 E-06$ & $4.828 E-06$ & $1.892 E-06$ & $2.823 E-06$ & $7.841 E-06$ & $2.823 E-06$ \\
\hline IEFM [42] & $8.789 E-06$ & $1.973 E-05$ & $8.788 E-06$ & $1.284 E-05$ & $3.278 E-05$ & $1.284 E-05$ & $1.902 E-05$ & $5.285 E-05$ & $1.902 E-05$ \\
\hline
\end{tabular}

TABLE 3: Comparison of absolute errors of GBH equation at $\mu=3.9, \Delta t=0.0001$ taking different values of $\delta$ for case 1 .

\begin{tabular}{lccccrr}
\hline$t=0.8$ & \multicolumn{3}{c}{$\delta=1$} & \multicolumn{3}{c}{$\delta=8$} \\
$x$ & 0.1 & 0.5 & 0.9 & 0.1 & 0.5 & 0.9 \\
\hline HBSCM & $5.870 E-09$ & $1.342 E-08$ & $5.870 E-09$ & $6.805 E-06$ & $1.555 E-05$ & $6.800 E-06$ \\
HWM [46] & $1.586 E-08$ & $4.662 E-08$ & $1.586 E-08$ & $1.404 E-05$ & $4.123 E-05$ & $1.404 E-05$ \\
\hline
\end{tabular}

TABLE 4: Comparison of maximum errors of GBH equation at $\mu=3.9, \Delta t=0.00005$ taking different values of $\delta$ for case 1 .

\begin{tabular}{lccr}
\hline$t=1.0$ & $\delta=1$ & $\delta=4$ & $\delta=8$ \\
\hline HBSCM & $1.342 E-08$ & $5.325 E-06$ & $1.555 E-05$ \\
NDDA [22] & $4.685 E-08$ & $1.532 E-05$ & $4.141 E-05$ \\
\hline
\end{tabular}

TABLE 5: Comparison of absolute errors calculated by HBSCM with the existing method at $\Delta t=0.01, \mu=2.6$ for case 1 .

\begin{tabular}{|c|c|c|c|c|c|c|c|c|c|}
\hline \multirow[b]{2}{*}{$x$} & \multicolumn{3}{|c|}{$t=0.05$} & \multicolumn{3}{|c|}{$t=0.1$} & \multicolumn{3}{|c|}{$t=1.0$} \\
\hline & 0.1 & 0.5 & 0.9 & 0.1 & 0.5 & 0.9 & 0.1 & 0.5 & 0.9 \\
\hline \multicolumn{10}{|c|}{$\delta=1$} \\
\hline HBSCM & $8.48 E-11$ & $1.91 E-10$ & $8.58 E-11$ & $1.23 E-10$ & $3.18 E-10$ & $1.25 E-10$ & $1.87 E-10$ & $5.22 E-10$ & $1.88 E-10$ \\
\hline LRBDQ [58] & $7.97 E-10$ & $1.87 E-08$ & $3.83 E-08$ & $1.59 E-09$ & $3.75 E-08$ & $7.66 E-08$ & $1.60 E-08$ & $3.75 E-07$ & $7.65 E-07$ \\
\hline \multicolumn{10}{|c|}{$\delta=2$} \\
\hline HBSCM & $1.14 E-08$ & $2.57 E-08$ & $1.14 E-08$ & $1.67 E-08$ & $4.26 E-08$ & $1.66 E-08$ & $2.44 E-08$ & $6.77 E-08$ & $2.43 E-08$ \\
\hline LRBDQ [58] & $1.15 E-09$ & $8.70 E-07$ & $1.75 E-06$ & $2.26 E-09$ & $1.75 E-06$ & $3.50 E-06$ & $1.37 E-08$ & $1.75 E-05$ & $3.49 E-05$ \\
\hline \multicolumn{10}{|c|}{$\delta=3$} \\
\hline HBSCM & $7.69 E-08$ & $1.72 E-07$ & $7.65 E-08$ & $1.11 E-07$ & $2.85 E-07$ & $1.11 E-07$ & $1.63 E-07$ & $4.54 E-07$ & $1.63 E-07$ \\
\hline LRBDQ [58] & $9.40 E-07$ & $3.19 E-06$ & $7.30 E-06$ & $1.89 E-06$ & $6.83 E-06$ & $1.47 E-05$ & $1.89 E-05$ & $6.38 E-05$ & $1.46 E-04$ \\
\hline
\end{tabular}


TABLE 6: Comparison of error norms at $\alpha=0.001, \beta=0.001, \gamma=0.001, \Delta t=0.0001, \epsilon=1, \delta=2$ for case 2.

\begin{tabular}{lcccr}
\hline$t$ & & HBSCM & \multicolumn{2}{c}{ LRBF [58] } \\
\\
\hline 0.0005 & $L_{\infty}$ & $L_{2}$ & $L_{\infty}$ & $1.3610 E-11$ \\
0.001 & $5.9337 E-13$ & $8.8011 E-14$ & $2.2152 E-11$ & $2.7153 E-11$ \\
0.5 & $1.1867 E-12$ & $2.3285 E-13$ & $4.4301 E-11$ & $1.3565 E-08$ \\
1 & $1.4722 E-10$ & $8.7302 E-10$ & $2.2152 E-08$ & $2.7130 E-08$ \\
5 & $1.4831 E-10$ & $1.3634 E-09$ & $4.4304 E-08$ & $1.3536 E-07$ \\
\hline
\end{tabular}

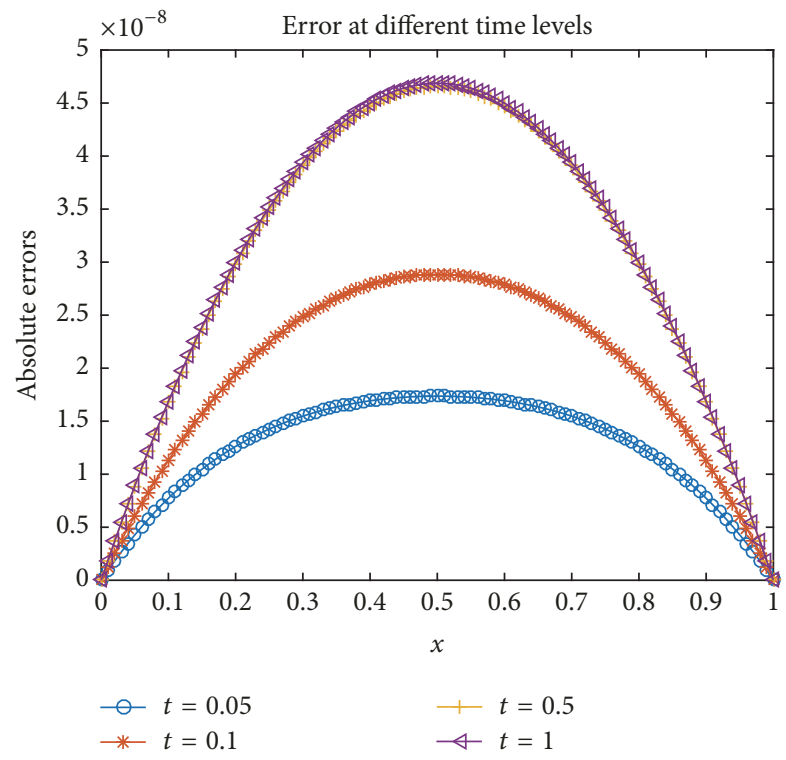

FIGURE 2: Absolute errors at different time levels when $\Delta t=0.0001$, $\mu=3.9, \delta=1$ for case 1 .

absolute errors can be visualized in the figures and tables due to the hybrid parameter. It is pertinent to claim that the proposed method provides accurate and improves results as compared to others.

(2) When $\alpha=0.001, \beta=0.001, \gamma=0.001, \Delta t=0.0001$, $\epsilon=1, \delta=2$, GBH equation can be [58] described as follows:

$$
\begin{aligned}
u_{t} & +0.001 u^{2} u_{x}-u_{x x} \\
& =0.001 u\left(1-u^{2}\right)\left(u^{2}-0.001\right) .
\end{aligned}
$$

In Table 6, the error norms are calculated at different time levels for $\Delta t=0.0001$ and compared with LRBFDQ [58]. Figures 4 and 5 depict the error norms at different time levels. It can be concluded that the proposed method is more accurate than LRBFDQ [58]

(3) When $\alpha=0.1, \beta=0.001, \gamma=0.0001, \epsilon=1$, GBH equation is expressed as follows:

$$
u_{t}+0.1 u^{\delta} u_{x}-u_{x x}=0.001 u\left(1-u^{\delta}\right)\left(u^{\delta}-0.0001\right) \text {. }
$$

A comparison of the absolute errors calculated by HBSCM at $\delta=1,2,8$ is presented in Table 7 with the

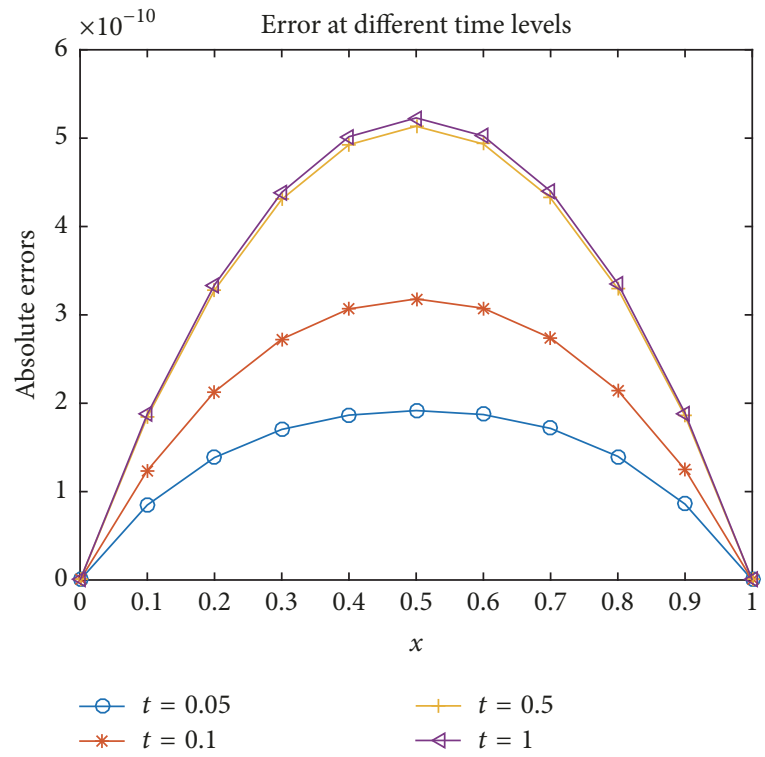

Figure 3: Absolute errors at different time levels when $\Delta t=0.01$, $\mu=2.6, \delta=1$ for case 1 .

existing methods named spectral collocation method (SCM) [20] and IEFM [42]. The order of convergence calculated numerically by HBSCM is compared with IEFM [42] in Table 8. It can be concluded that present method has a rapid convergence. Figure 6 depicts the absolute errors which clearly demonstrate that increase in time does not disturb the accuracy of the obtained results

(4) When $\alpha=0, \beta=1, \gamma=0.001, \mu=1.65, \Delta t=0.001$, $\epsilon=1, \mathrm{GBH}$ can be represented as follows:

$$
u_{t}-u_{x x}=u\left(1-u^{\delta}\right)\left(u^{\delta}-0.001\right) .
$$

In Table 9, we tabulate a comparison between HBSCM and the existing methods CFDS [26], FONS [33], ADM [38], Galerkin method (GM) [44], higher order finite difference method (HFDM) [52], MCBS [55], cubic B-spline algorithm (CBSA) [56], and optimal homotopy asymptotic method (OHAM) [69] at $\delta=1,3$. Computations of the absolute errors and two different types of error norms have been mentioned in Tables 10 and 11, respectively. Figure 7 exhibits the computed results at different time levels 
TABLE 7: Comparison of absolute errors at different values of $\delta$ and parameter $\mu=1.9$ for case 3 .

\begin{tabular}{|c|c|c|c|c|c|c|c|c|c|}
\hline \multirow[b]{2}{*}{$x$} & \multicolumn{3}{|c|}{$t=0.2$} & \multicolumn{3}{|c|}{$t=0.5$} & \multicolumn{3}{|c|}{$t=0.8$} \\
\hline & 0.1 & 0.5 & 0.9 & 0.1 & 0.5 & 0.9 & 0.1 & 0.5 & 0.9 \\
\hline \multicolumn{10}{|c|}{$\delta=1$} \\
\hline HBSCM & $1.315 E-13$ & $3.569 E-13$ & $1.315 E-13$ & $1.490 E-13$ & $4.135 E-13$ & $1.490 E-13$ & $1.499 E-13$ & $4.165 E-13$ & $1.499 E-13$ \\
\hline SCM [20] & - & - & - & $8.381 E-12$ & $4.879 E-11$ & $2.859 E-11$ & - & - & - \\
\hline IEFM [42] & $1.561 E-13$ & $4.238 E-13$ & $1.561 E-13$ & $1.770 E-13$ & $4.911 E-13$ & $1.770 E-13$ & $1.780 E-13$ & $4.946 E-13$ & $1.780 E-13$ \\
\hline \multicolumn{10}{|c|}{$\delta=2$} \\
\hline HBSCM & $1.928 E-11$ & $5.232 E-11$ & $1.928 E-11$ & $2.1847 E-11$ & $6.062 E-11$ & $2.184 E-11$ & $2.198 E-11$ & $6.105 E-11$ & $2.198 E-11$ \\
\hline SCM [20] & - & - & - & $5.554 E-10$ & $3.815 E-09$ & $2.236 E-09$ & - & - & - \\
\hline IEFM [42] & $3.838 E-11$ & $1.041 E-10$ & $3.838 E-11$ & $4.349 E-11$ & $1.206 E-10$ & $4.349 E-11$ & $4.375 E-11$ & $1.215 E-10$ & $4.375 E-11$ \\
\hline \multicolumn{10}{|c|}{$\delta=8$} \\
\hline HBSCM & $8.877 E-10$ & $2.408 E-09$ & $8.877 E-10$ & $1.005 E-09$ & $2.790 E-09$ & $1.005 E-09$ & $1.011 E-09$ & $2.810 E-09$ & $1.011 E-09$ \\
\hline SCM [20] & - & - & - & $3.395 E-08$ & $1.976 E-07$ & $1.158 E-07$ & - & - & - \\
\hline IEFM [42] & $1.743 E-08$ & $4.732 E-08$ & $1.743 E-08$ & $1.976 E-08$ & $5.483 E-08$ & $1.976 E-08$ & $1.988 E-08$ & $5.522 E-08$ & $1.988 E-08$ \\
\hline
\end{tabular}

TABLE 8: Order of convergence $(p)$ at $t=1$ for case 3.

\begin{tabular}{lcccccc}
\hline$n$ & 2 & 4 & 8 & 16 & 32 & 64 \\
\hline$L_{\infty}$ & $3.054 E-06$ & $7.008 E-07$ & $1.761 E-07$ & $4.874 E-08$ & $1.710 E-08$ & $9.212 E-09$ \\
HBSCM $(p)$ & - & 2.123 & 1.992 & 1.853 & 1.510 & 0.892 \\
IEFM $[42]$ & - & 0.175608438 & 0.078691220 & 0.041403087 & 0.021266315 & 0.011014546 \\
\hline
\end{tabular}

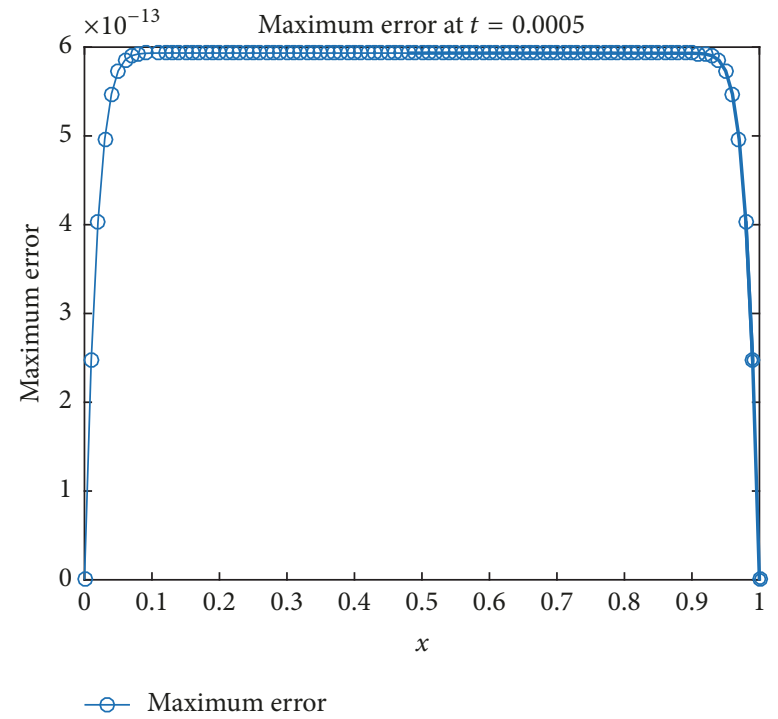

Figure 4: $L_{\infty}$ errors at $\delta=2, t=0.0005$ for case 2 .

which enable us to claim that the proposed scheme is superior to the existing method in the terms of accuracy.

(5) When $\alpha=5, \delta=2, \beta=10, \mu=1.6, \Delta t=0.0001$, $\epsilon=1$, the following $\mathrm{GBH}$ can be obtained:

$$
u_{t}+5 u^{2} u_{x}-u_{x x}=10 u\left(1-u^{2}\right)\left(u^{2}-\gamma\right) \text {. }
$$

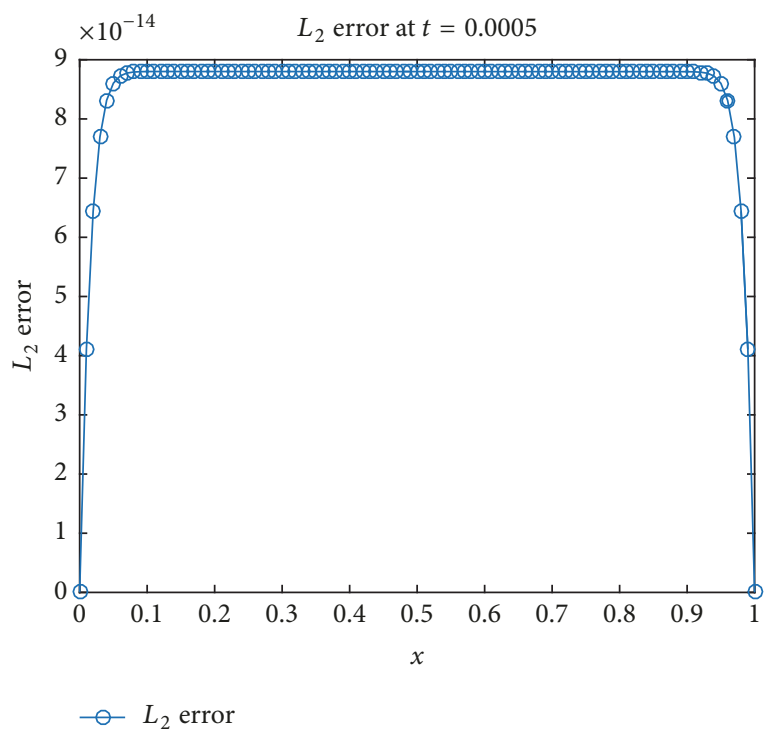

FIgURE 5: $L_{2}$ errors at $\delta=2, t=0.0005$ for case 2 .

The absolute errors are recorded in Table 12 at different values of $\gamma=10^{-3}, 10^{-4}, 10^{-5}$, calculated by HBSCM and compared with IEFM [42]. Figure 8 explains these errors graphically at $\gamma=10^{-3}$. A prominent difference in the accuracy can be seen in the figure and table due to the hybrid parameter. 
TABLE 9: Comparison of absolute errors computed by HBSCM with other methods at $\alpha=0, \beta=1, \gamma=0.001$ for case 4 .

\begin{tabular}{|c|c|c|c|c|c|c|c|c|c|}
\hline \multirow[b]{2}{*}{$x$} & \multicolumn{3}{|c|}{$t=0.05$} & \multicolumn{3}{|c|}{$t=0.1$} & \multicolumn{3}{|c|}{$t=1.0$} \\
\hline & 0.1 & 0.5 & 0.9 & 0.1 & 0.5 & 0.9 & 0.1 & 0.5 & 0.9 \\
\hline \multicolumn{10}{|c|}{$\delta=1$} \\
\hline HBSCM & $1.030 E-08$ & $2.313 E-08$ & $1.030 E-08$ & $1.506 E-08$ & $3.844 E-08$ & $1.506 E-08$ & $2.248 E-08$ & $6.246 E-08$ & $2.248 E-08$ \\
\hline CFDS [26] & $1.026 E-08$ & $2.304 E-08$ & $1.026 E-08$ & $1.502 E-08$ & $3.832 E-08$ & $1.502 E-08$ & $2.248 E-08$ & $6.246 E-08$ & $2.248 E-08$ \\
\hline FONS [33] & $2.498 E-08$ & $2.498 E-08$ & $2.498 E-08$ & $4.997 E-08$ & $4.997 E-08$ & $4.997 E-08$ & $4.997 E-07$ & $4.997 E-07$ & $4.997 E-07$ \\
\hline ADM [40] & $1.874 E-07$ & $1.874 E-07$ & $1.875 E-07$ & $3.749 E-07$ & $3.749 E-07$ & $3.750 E-07$ & $3.750 E-07$ & $3.750 E-07$ & $3.750 E-07$ \\
\hline GM [44] & $1.069 E-08$ & $9.259 E-09$ & $7.892 E-09$ & $2.318 E-08$ & $2.174 E-08$ & $2.038 E-08$ & $2.487 E-07$ & $2.472 E-07$ & $2.459 E-07$ \\
\hline HFDM [52] & $1.030 E-08$ & $2.313 E-08$ & $1.030 E-08$ & $1.506 E-08$ & $3.844 E-08$ & $1.506 E-08$ & $2.248 E-08$ & $6.246 E-08$ & $2.248 E-08$ \\
\hline MCBS [55] & $1.004 E-08$ & $2.304 E-08$ & $1.004 E-08$ & $1.479 E-08$ & $3.825 E-08$ & $1.479 E-08$ & $2.220 E-08$ & $6.216 E-08$ & $2.220 E-08$ \\
\hline CBSA [56] & $1.029 E-08$ & $2.313 E-08$ & $1.029 E-08$ & $1.502 E-08$ & $3.843 E-08$ & $1.502 E-08$ & $2.248 E-08$ & $6.246 E-08$ & $2.248 E-08$ \\
\hline $\mathrm{OH}$ & $2.498 E-08$ & $.498 E-08$ & $2.498 E-08$ & $4.997 E-08$ & $4.997 E-08$ & $4.997 E-08$ & $4.997 E-07$ & $4.997 E-07$ & $4.997 E-07$ \\
\hline \multicolumn{10}{|c|}{$\delta=3$} \\
\hline HBSCM & $1.635 E-06$ & $3.673 E-06$ & $1.635 E-06$ & $2.391 E-06$ & $6.102 E-06$ & $2.390 E-06$ & $3.567 E-06$ & $9.907 E-06$ & $3.566 E-06$ \\
\hline CFDS [26] & $1.630 E-06$ & $3.658 E-06$ & $1.629 E-06$ & $2.385 E-06$ & $6.083 E-06$ & $2.385 E-06$ & $3.567 E-06$ & $9.907 E-06$ & $3.566 E-06$ \\
\hline FONS [33] & $3.967 E-06$ & $3.966 E-06$ & $3.965 E-06$ & $7.934 E-06$ & $7.933 E-06$ & $7.931 E-06$ & $7.934 E-06$ & $7.933 E-06$ & $7.931 E-06$ \\
\hline $\mathrm{ADM}[40]$ & $1.984 E-06$ & $1.983 E-06$ & $1.983 E-06$ & $3.968 E-06$ & $3.967 E-06$ & $3.966 E-06$ & $3.966 E-06$ & $3.965 E-06$ & $3.964 E-06$ \\
\hline HFDM [52] & $1.635 E-06$ & $3.672 E-06$ & $1.635 E-06$ & $2.391 E-06$ & $6.101 E-06$ & $2.390 E-06$ & $3.567 E-06$ & $9.907 E-06$ & $3.566 E-06$ \\
\hline MCBS [55] & $1.594 E-06$ & $3.658 E-06$ & $1.594 E-06$ & $2.347 E-06$ & $6.072 E-06$ & $2.347 E-06$ & $3.522 E-06$ & $9.861 E-06$ & $3.521 E-06$ \\
\hline CBSA [56] & $1.634 E-06$ & $3.672 E-06$ & $1.634 E-06$ & $2.390 E-06$ & $6.101 E-06$ & $2.390 E-06$ & $3.567 E-06$ & $9.907 E-06$ & $3.566 E-06$ \\
\hline
\end{tabular}

TABLE 10: Comparison of absolute errors calculated by HBSCM with the existing method at $\Delta t=0.01, \mu=2.6$ for case 4 .

\begin{tabular}{|c|c|c|c|c|c|c|c|c|c|}
\hline \multirow[b]{2}{*}{$x$} & \multicolumn{3}{|c|}{$t=0.05$} & \multicolumn{3}{|c|}{$t=0.1$} & \multicolumn{3}{|c|}{$t=1.0$} \\
\hline & 0.1 & 0.5 & 0.9 & 0.1 & 0.5 & 0.9 & 0.1 & 0.5 & 0.9 \\
\hline \multicolumn{10}{|c|}{$\delta=1$} \\
\hline HBSCM & $2.50 E-09$ & $5.63 E-09$ & $2.50 E-09$ & $3.65 E-09$ & $9.33 E-09$ & $3.65 E-09$ & $5.41 E-09$ & $1.50 E-08$ & $5.41 E-09$ \\
\hline LRBDQ [58] & $1.37 E-09$ & $2.50 E-08$ & $1.37 E-09$ & $2.70 E-09$ & $5.00 E-08$ & $2.70 E-09$ & $2.70 E-08$ & $5.00 E-07$ & $2.70 E-08$ \\
\hline \multicolumn{10}{|c|}{$\delta=2$} \\
\hline HBSCM & $1.1 E-07$ & $2.5 E-07$ & $1.1 E-07$ & $1.6 E-07$ & $4.1 E-07$ & $1.6 E-07$ & $2.4 E-07$ & $6.7 E-07$ & $2.4 E-07$ \\
\hline LRBDQ [58] & $6.1 E-08$ & $1.1 E-06$ & $6.1 E-08$ & $1.2 E-07$ & $2.2 E-06$ & $1.2 E-07$ & $1.1 E-06$ & $2.2 E-05$ & $1.2 E-06$ \\
\hline \multicolumn{10}{|c|}{$\delta=3$} \\
\hline HBSCM & $3.98 E-07$ & $8.93 E-07$ & $3.97 E-07$ & $5.79 E-07$ & $1.48 E-06$ & $5.79 E-07$ & $8.56 E-07$ & $2.37 E-06$ & $8.55 E-07$ \\
\hline LRBDQ [58] & $2.17 E-07$ & $3.97 E-06$ & $2.19 E-07$ & $4.33 E-07$ & $7.93 E-06$ & $4.37 E-07$ & $4.32 E-06$ & $7.93 E-05$ & $4.36 E-06$ \\
\hline
\end{tabular}

TABLE 11: Comparison of error norms for $\alpha=0, \beta=1, \gamma=0.001, \Delta t=0.01, \epsilon=1, \delta=2$ of case 4 .

\begin{tabular}{lcccc}
\hline$t$ & HBSCM & \multicolumn{2}{c}{ LRBF [58] } & $L_{\infty}$ \\
\hline 0.05 & $L_{\infty}$ & $L_{2}$ & $5.1347 E-08$ & $3.0711 E-08$ \\
0.1 & $2.5183 E-07$ & $1.2105 E-07$ & $1.0269 E-07$ & $6.1418 E-08$ \\
1 & $4.1746 E-07$ & $2.8221 E-07$ & $1.0262 E-06$ & $6.1359 E-07$ \\
5 & $6.7151 E-07$ & $1.9621 E-06$ & $5.1155 E-06$ & $3.0649 E-06$ \\
50 & $1.3669 E-06$ & $4.6487 E-06$ & $4.9458 E-05$ & $3.0125 E-05$ \\
\hline
\end{tabular}

(6) When $\alpha=1, \delta=1, \gamma=0.0001, \mu=1.35, \Delta t=0.0001$, $\epsilon=1, \mathrm{GBH}$ equation is

$$
u_{t}+u u_{x}-u_{x x}=\beta u(1-u)(u-0.0001) .
$$

The computed results by HBSCM at $\beta=1,10,100$ are recorded in Table 13 and compared with the existing method IEFM [42]. Figure 9 illustrates the behaviour of these errors graphically at $\beta=1$. 
TABLE 12: Comparison of absolute errors obtained by HBSCM with existing methods at different values of $\gamma$ for case 5 .

\begin{tabular}{|c|c|c|c|c|c|c|c|c|c|}
\hline \multirow[b]{2}{*}{$x$} & \multicolumn{3}{|c|}{$t=0.2$} & \multicolumn{3}{|c|}{$t=0.5$} & \multicolumn{3}{|c|}{$t=0.8$} \\
\hline & 0.1 & 0.5 & 0.9 & 0.1 & 0.5 & 0.9 & 0.1 & 0.5 & 0.9 \\
\hline \multicolumn{10}{|c|}{$\gamma=10^{-3}$} \\
\hline HBSCM & $6.230 E-06$ & $1.690 E-06$ & $6.231 E-06$ & $7.056 E-06$ & $1.958 E-05$ & $7.057 E-06$ & $7.094 E-06$ & $1.970 E-05$ & $7.095 E-06$ \\
\hline IEFM [42] & $2.075 E-05$ & $5.633 E-05$ & $2.076 E-05$ & $2.339 E-05$ & $6.495 E-05$ & $2.340 E-05$ & $2.340 E-05$ & $6.503 E-05$ & $2.341 E-05$ \\
\hline \multicolumn{10}{|c|}{$\gamma=10^{-4}$} \\
\hline HBSCM & $1.971 E-07$ & $5.350 E-07$ & $1.971 E-07$ & $2.233 E-07$ & $6.198 E-07$ & $2.233 E-07$ & $2.247 E-07$ & $6.242 E-07$ & $2.247 E-07$ \\
\hline IEFM [42] & $6.580 E-07$ & $1.785 E-06$ & $6.580 E-07$ & $7.453 E-07$ & $2.068 E-06$ & $7.453 E-07$ & $7.494 E-07$ & $2.081 E-06$ & $7.495 E-07$ \\
\hline \multicolumn{10}{|c|}{$\gamma=10^{-5}$} \\
\hline HBSCM & $6.235 E-09$ & $1.692 E-08$ & $6.235 E-09$ & $7.065 E-09$ & $1.960 E-08$ & $7.065 E-09$ & $7.108 E-09$ & $1.974 E-08$ & $7.108 E-09$ \\
\hline IEFM [42] & $2.081 E-08$ & $5.648 E-08$ & $2.081 E-08$ & $2.358 E-08$ & $6.545 E-08$ & $2.358 E-08$ & $2.372 E-08$ & $6.591 E-08$ & $2.373 E-08$ \\
\hline
\end{tabular}

TABLE 13: Comparison of absolute errors by HBSCM with other methods at $\alpha=1, \gamma=10^{-4}, \delta=1$ for case 6.

\begin{tabular}{|c|c|c|c|c|c|c|c|c|c|}
\hline \multirow[b]{2}{*}{$x$} & \multicolumn{3}{|c|}{$t=0.2$} & \multicolumn{3}{|c|}{$t=0.5$} & \multicolumn{3}{|c|}{$t=0.8$} \\
\hline & 0.1 & 0.5 & 0.9 & 0.1 & 0.5 & 0.9 & 0.1 & 0.5 & 0.9 \\
\hline \multicolumn{10}{|c|}{$\beta=1$} \\
\hline HBSCM & $1.479 E-10$ & $4.015 E-10$ & $1.479 E-10$ & $1.676 E-10$ & $4.653 E-10$ & $1.676 E-10$ & $1.687 E-10$ & $4.686 E-10$ & $1.687 E-10$ \\
\hline IEFM [42] & $2.959 E-10$ & $8.029 E-10$ & $2.959 E-10$ & $3.353 E-10$ & $9.304 E-10$ & $3.353 E-10$ & $3.373 E-10$ & $9.370 E-10$ & $3.373 E-10$ \\
\hline \multicolumn{10}{|c|}{$\beta=10$} \\
\hline HBSCM & $1.775 E-09$ & $4.818 E-09$ & $1.775 E-09$ & $2.012 E-09$ & $5.583 E-09$ & $2.012 E-09$ & $2.024 E-09$ & $5.622 E-09$ & $2.024 E-09$ \\
\hline IEFM [42] & $4.142 E-09$ & $1.124 E-08$ & $4.142 E-09$ & $4.694 E-09$ & $1.302 E-08$ & $4.694 E-09$ & $4.723 E-09$ & $1.311 E-08$ & $4.723 E-09$ \\
\hline \multicolumn{10}{|c|}{$\beta=100$} \\
\hline HBSCM & $1.905 E-08$ & $5.171 E-08$ & $1.905 E-08$ & $2.159 E-08$ & $5.991 E-08$ & $2.159 E-08$ & $2.172 E-08$ & $6.034 E-08$ & $2.172 E-08$ \\
\hline IEFM [42] & $4.662 E-08$ & $1.265 E-07$ & $4.662 E-08$ & $5.283 E-08$ & $1.466 E-07$ & $5.283 E-08$ & $5.314 E-08$ & $1.476 E-07$ & $5.314 E-08$ \\
\hline
\end{tabular}

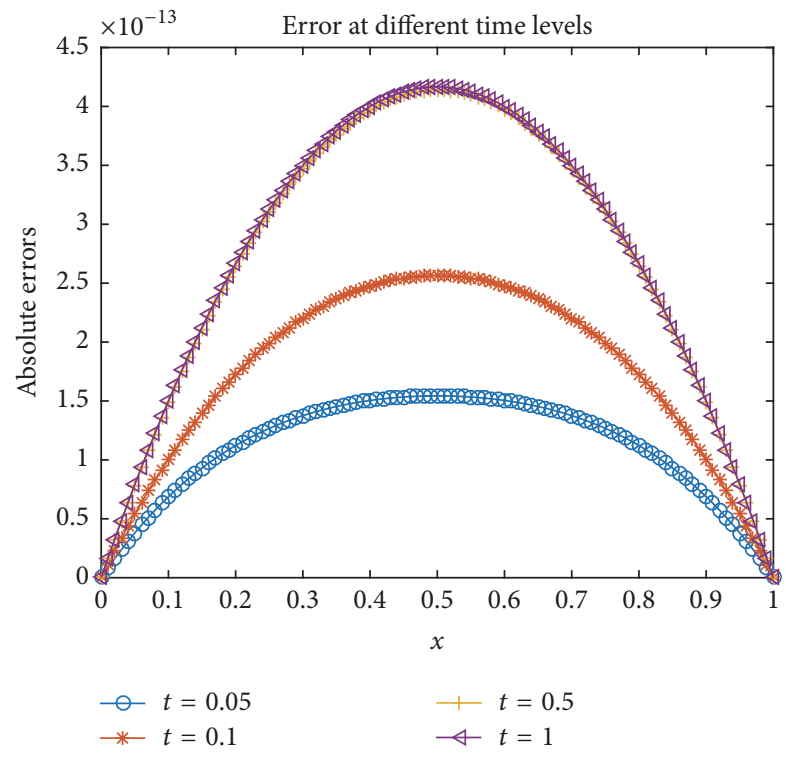

FIGURE 6: Absolute errors at different time levels when $\delta=1$ for case 3.

\subsection{Numerical Test Cases for Model II}

(7) When $\alpha=1, \beta=1, \Delta t=0.00001, \epsilon=1, \delta=1$, GBF equation in the domain $[-1,1]$ can be written as follows:

$$
u_{t}+u u_{x}-u_{x x}=u(1-u) .
$$

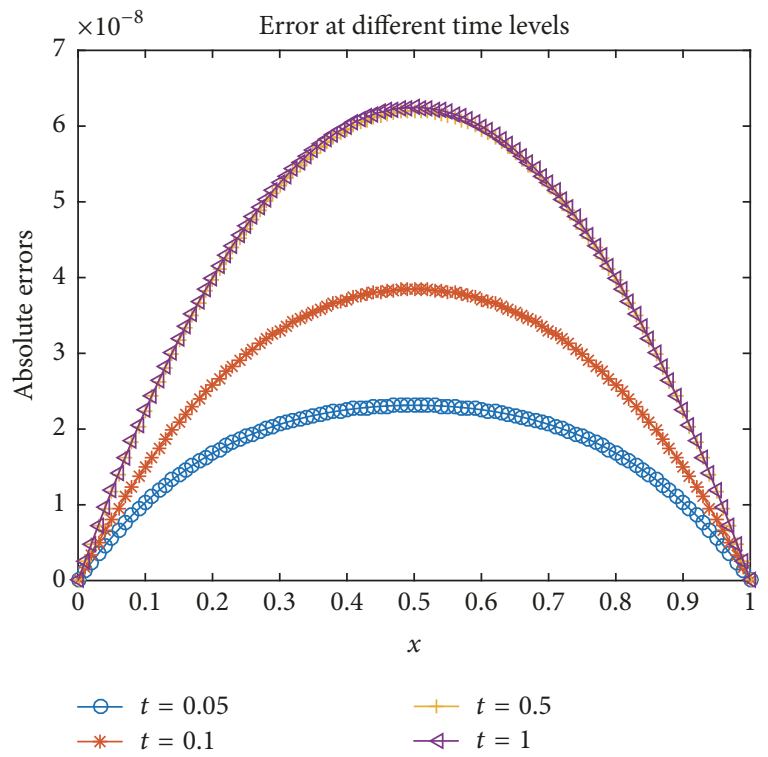

Figure 7: Absolute errors at $\alpha=0, \beta=1, \gamma=0.001, \delta=1$ for case 4.

In Table 14, we record the absolute errors obtained by HBSCM for three different values of hybrid parameter $\mu$ and compare them with the cubic B-spline method (CBSM) [53]. Table 15 establishes a comparison of error norms $L_{2}$ and $L_{\infty}$ calculated by HBSCM at 
TABLE 14: Comparison of absolute errors with [53] $\alpha=1, \beta=1, \delta=1$ for case 7 .

\begin{tabular}{|c|c|c|c|c|c|}
\hline$x$ & $t$ & $\mu=1.1$ & $\mu=1.5$ & $\mu=1.9$ & CBSM [53] \\
\hline \multirow{4}{*}{-0.5} & 0.5 & $3.142 E-06$ & $1.463 E-06$ & $4.226 E-07$ & $6.108 E-06$ \\
\hline & 1.0 & $3.408 E-06$ & $1.177 E-06$ & $4.825 E-07$ & $5.496 E-06$ \\
\hline & 2.0 & $4.505 E-06$ & $1.766 E-06$ & $5.298 E-07$ & $3.758 E-06$ \\
\hline & 4.0 & $4.149 E-06$ & $3.644 E-06$ & $7.470 E-07$ & $6.071 E-07$ \\
\hline \multirow{4}{*}{0.0} & 0.5 & $4.329 E-06$ & $2.196 E-06$ & $5.588 E-07$ & $3.072 E-06$ \\
\hline & 1.0 & $4.495 E-06$ & $1.621 E-06$ & $6.447 E-07$ & $4.118 E-06$ \\
\hline & 2.0 & $5.650 E-06$ & $2.109 E-06$ & $6.744 E-07$ & $4.559 E-06$ \\
\hline & 4.0 & $6.247 E-06$ & $4.725 E-06$ & $9.711 E-07$ & $3.117 E-07$ \\
\hline \multirow{4}{*}{0.5} & 0.5 & $3.514 E-06$ & $1.893 E-06$ & $1.339 E-07$ & $1.159 E-06$ \\
\hline & 1.0 & $3.437 E-06$ & $1.279 E-06$ & $4.973 E-07$ & $3.680 E-06$ \\
\hline & 2.0 & $4.175 E-06$ & $1.499 E-06$ & $5.035 E-07$ & $5.724 E-06$ \\
\hline & 4.0 & $6.525 E-06$ & $3.595 E-06$ & $7.400 E-07$ & $1.672 E-07$ \\
\hline \multirow{4}{*}{0.9} & 0.5 & $9.634 E-07$ & $5.331 E-07$ & $0.456 E-07$ & $1.315 E-06$ \\
\hline & 1.0 & $8.952 E-07$ & $3.360 E-07$ & $1.303 E-07$ & $4.758 E-06$ \\
\hline & 2.0 & $1.081 E-06$ & $3.814 E-07$ & $1.308 E-07$ & $7.098 E-06$ \\
\hline & 4.0 & $9.281 E-06$ & $9.499 E-07$ & $1.950 E-07$ & $2.364 E-07$ \\
\hline
\end{tabular}

TABLE 15: Comparison of error norms $L_{\infty}$ and $L_{2}$ calculated by HBSCM at $\mu=1.9$ with [53] for case 7 .

\begin{tabular}{lcccc}
\hline$T$ & & & & CBSM [53] \\
& HBSCM & $L_{\infty}$ & $L_{\infty}$ & $L_{2}$ \\
\hline 0.5 & $1.343 E-07$ & $1.750 E-07$ & $9.172 E-06$ & $6.953 E-06$ \\
1.0 & $1.347 E-07$ & $1.688 E-07$ & $6.852 E-06$ & $6.131 E-06$ \\
2.0 & $1.345 E-07$ & $1.059 E-07$ & $7.304 E-06$ & $7.228 E-06$ \\
4.0 & $1.593 E-07$ & $9.761 E-08$ & $6.443 E-07$ & $6.702 E-07$ \\
\hline
\end{tabular}

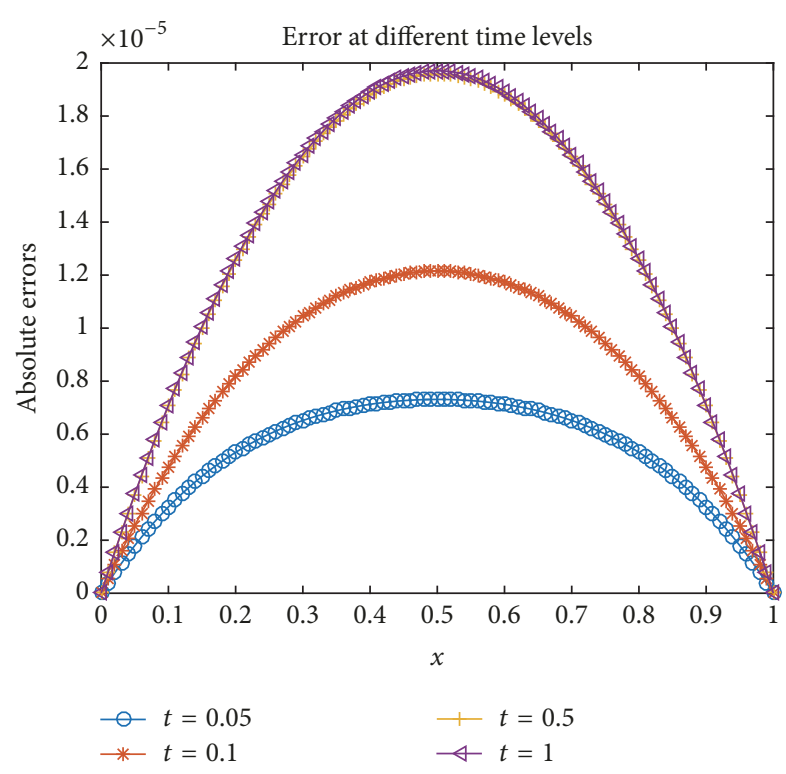

FIGURE 8: Absolute errors at $\alpha=5, \beta=10, \gamma=0.001, \delta=2$ for case 5 .

$\mu=1.5$ with CBSM [53]. The behaviour of the absolute errors is shown in Figure 10. The exact and approximate solutions are presented graphically in Figure 11 at different time levels. Figures 12 and 13 represent the pictorial view of $L_{2}$ and $L_{\infty}$ errors,

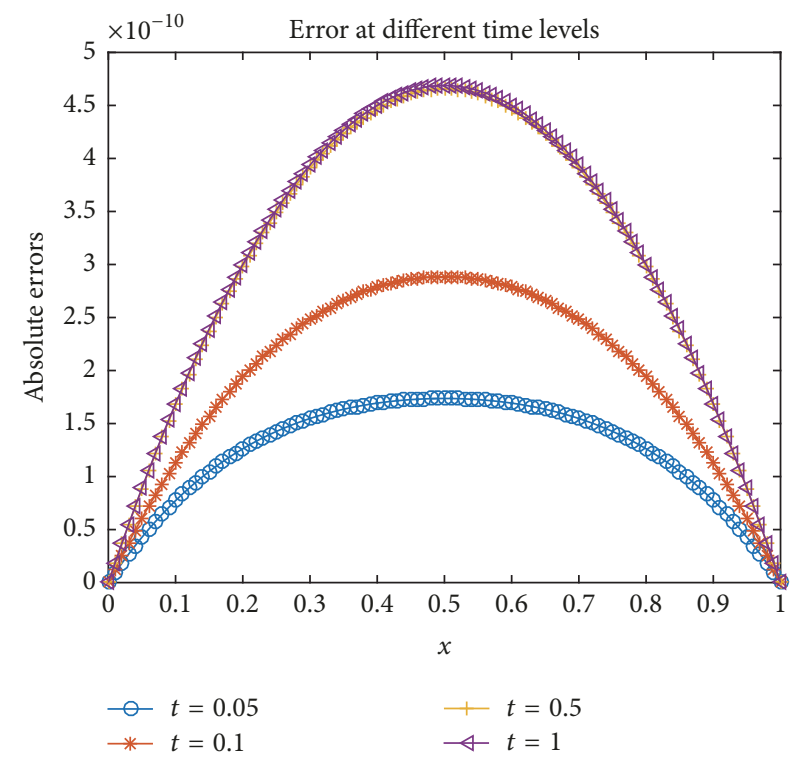

FIGURE 9: Absolute errors at $\alpha=1, \beta=1, \gamma=0.0001, \delta=1$ for case 6.

respectively. Figures 14 and 15 indicate that the proposed HBSCM approximates the exact solution quite nicely. It can be concluded from these figures and tables that the proposed method is more accurate than existing methods. 
TABLE 16: Comparison of absolute errors with existing methods at $\alpha=0.001, \beta=0.001, \delta=1$ for case 8 .

\begin{tabular}{lccccrr}
\hline & \multicolumn{3}{c}{$t=0.001$} & \multicolumn{3}{c}{$t=0.01$} \\
$x$ & 0.1 & 0.5 & 0.9 & 0.1 & 0.5 & 0.9 \\
\hline HBSCM & $3.056 E-10$ & $2.658 E-10$ & $3.055 E-10$ & $1.962 E-09$ & $2.659 E-09$ & $1.962 E-09$ \\
EFM[29] & $1.970 E-08$ & $3.580 E-09$ & $1.800 E-08$ & $1.970 E-08$ & $3.880 E-09$ & $1.740 E-08$ \\
IA [37] & $2.524 E-05$ & $2.524 E-05$ & $2.524 E-05$ & $5.999 E-10$ & $5.999 E-10$ & $5.999 E-10$ \\
ADM [40] & $1.937 E-06$ & $1.937 E-06$ & $1.937 E-06$ & $1.937 E-05$ & $1.937 E-05$ & $1.937 E-05$ \\
OHAM [69] & $2.250 E-08$ & $4.580 E-08$ & $4.580 E-08$ & $2.250 E-07$ & $4.580 E-07$ & $4.580 E-07$ \\
NAT [70] & $3.466 E-04$ & $9.870 E-03$ & $9.424 E-05$ & $1.178 E-05$ & $3.740 E-05$ & $6.378 E-08$ \\
\hline
\end{tabular}

TABLE 17: The absolute errors calculated by HBSCM and other methods at $\alpha=1, \beta=0, \delta=3$ for case 9.

\begin{tabular}{lcccccc}
\hline & & $t=0.0001$ & & \multicolumn{3}{c}{$t=0.001$} \\
$x$ & 0.1 & 0.5 & 0.9 & 0.1 & 0.5 & 0.9 \\
\hline HBSCM & $1.260 E-09$ & $5.543 E-10$ & $4.512 E-11$ & $1.147 E-08$ & $5.530 E-09$ & $5.148 E-10$ \\
CMM [27] & - & - & - & $1.900 E-05$ & $1.600 E-05$ & $1.500 E-05$ \\
EFM [29] & $4.550 E-07$ & $5.660 E-07$ & $7.000 E-07$ & $4.600 E-07$ & $5.610 E-07$ & $6.950 E-07$ \\
IA [37] & $8.780 E-08$ & $4.484 E-07$ & $4.175 E-07$ & $2.220 E-07$ & $2.635 E-07$ & $3.645 E-07$ \\
ADM [40] & $4.463 E-04$ & $1.860 E-03$ & $9.318 E-04$ & $4.439 E-04$ & $1.847 E-03$ & $9.042 E-04$ \\
NAT [70] & $4.780 E-04$ & $1.436 E-04$ & $7.659 E-05$ & $7.865 E-03$ & $5.323 E-04$ & $7.115 E-04$ \\
\hline
\end{tabular}

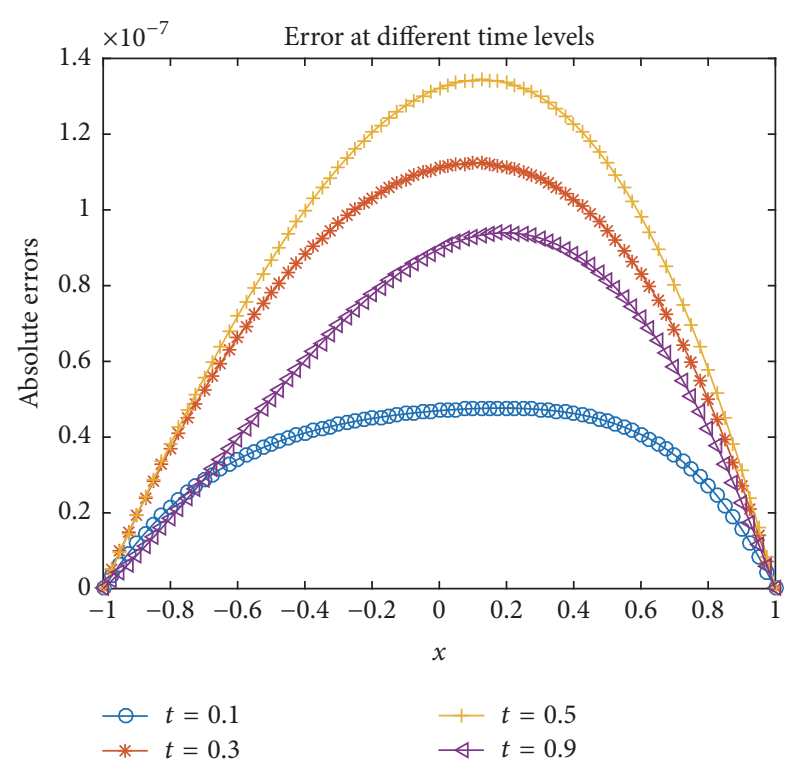

Figure 10: Absolute errors at $\alpha=1, \beta=1, \delta=1$ for case 7 .

(8) When $\alpha=0.001, \beta=0.001, \mu=1.3, \epsilon=1, \delta=1$, GBF equation in the domain $[0,1]$ can be described as

$$
u_{t}+0.001 u u_{x}-u_{x x}=0.001 u(1-u) .
$$

The absolute errors calculated at different time levels by proposed HBSCM are compared with the existing methods Exp-function method (EFM) [29],

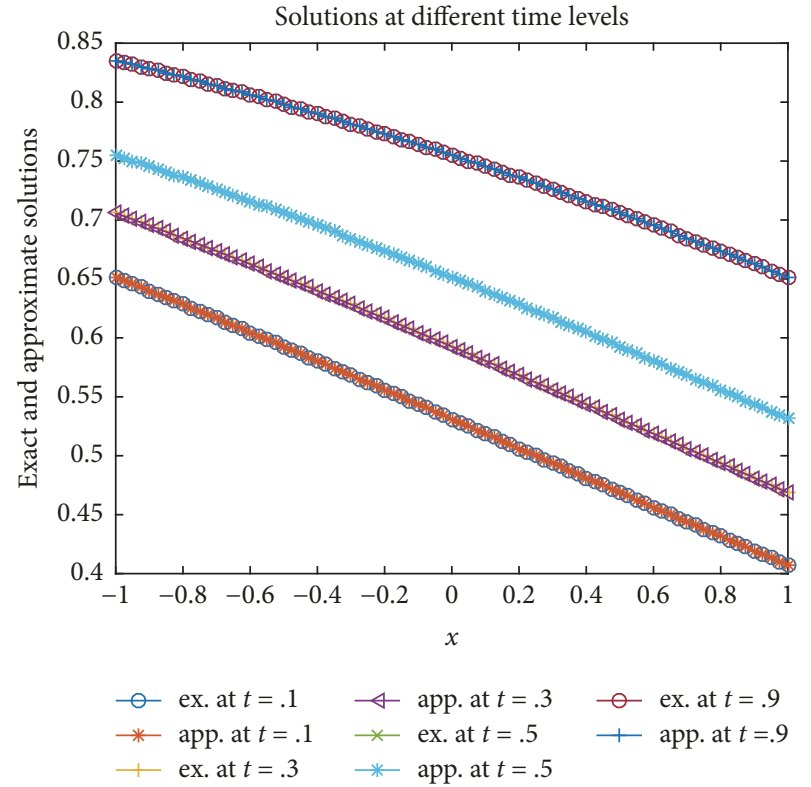

FIGURE 11: Exact and approximate solutions at $\alpha=1, \beta=1, \delta=1$ for case 7.

improved algorithm (IA) [37], ADM [40], OHAM [69], and Nonlinear Analytic Technique (NAT) [70] in Table 16. It is concluded that the proposed method is more accurate than these methods.

(9) When $\alpha=1, \beta=0, \Delta t=0.00001, \mu=1.4, \epsilon=1, \delta=$ $3, \mathrm{GBF}$ in the domain $[0,1]$ is represented as follows:

$$
u_{t}+u^{3} u_{x}-u_{x x}=0
$$




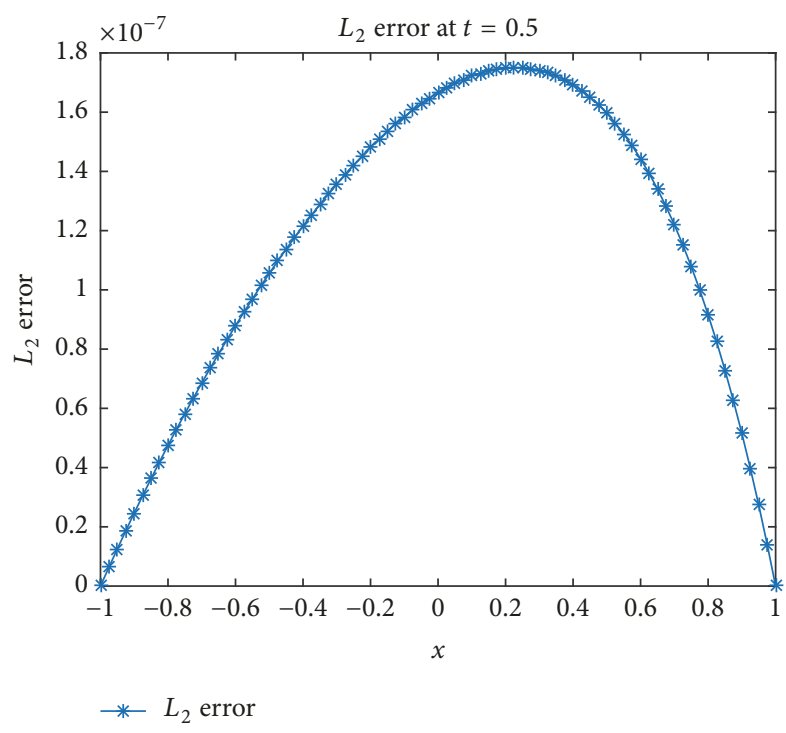

FIGURE 12: $L_{2}$ error norm at $\alpha=1, \beta=1, \delta=1$ for case 7 .

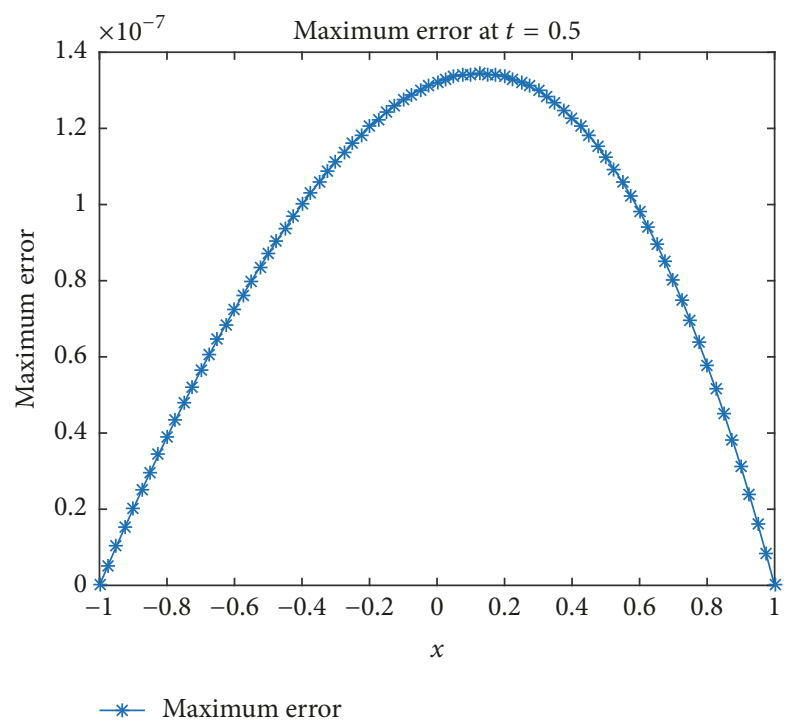

FIGURE 13: $L_{\infty}$ error norm at $\alpha=1, \beta=1, \delta=1$ for case 7 .

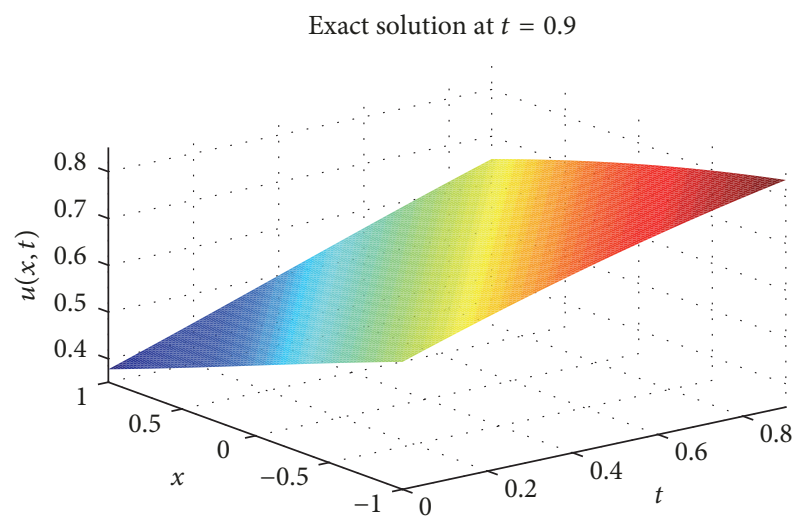

FIGURE 14: Exact solution in space at $\alpha=1, \beta=1, \delta=1, t=0.9$ for case 7.

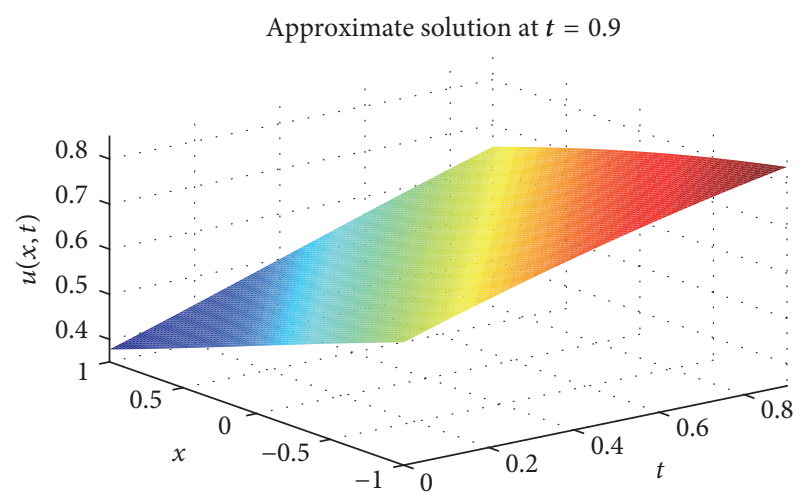

FIgURE 15: Approximate solution in space at $\alpha=1, \beta=1, \delta=1$, $t=0.9$ for case 7 .

The absolute errors are calculated by proposed HBSCM and compared with the existing methods: computational meshless method (CMM) [27], EFM [29], IA [37], ADM [40], and NAT [70] at two different time levels which are tabulated in Table 17. A remarkable difference in accuracy can be noted from this table.

\section{Concluding Remarks}

In this paper, a hybrid B-spline collocation method is developed for solving one-dimensional GBH and GBF equations with known initial and boundary conditions. A finite difference scheme and hybrid B-spline function are used to discretize the time and spatial derivatives, respectively. The hybrid B-spline method considered in this study is simple and straight forward by the application point of view. The obtained results are presented in Tables 1-17 and graphically shown in Figures 1-15. It is found that the numerical results are in excellent agreement with the analytical solutions. The proposed scheme is not only accurate but also quite different from the schemes [53-57] due to presence of parameter. The parameter $\mu>1$ provides the better approximation to the exact solution as compared to classical cubic B-spline function. The stability analysis using Von Neumann approach has also been presented. It is shown that the method is unconditionally stable for any step length.

\section{Conflicts of Interest}

The authors declare that they have no conflicts of interest.

\section{References}

[1] W. Hu, Z. Deng, S. Han, and W. Zhang, "Generalized multisymplectic integrators for a class of Hamiltonian nonlinear wave PDEs," Journal of Computational Physics, vol. 235, pp. 394-406, 2013.

[2] W. Hu, Z. Deng, B. Wang, and H. Ouyang, "Chaos in an embedded single-walled carbon nanotube," Nonlinear Dynamics, vol. 72, no. 1-2, pp. 389-398, 2013.

[3] W. Hu, Z. Deng, and Y. Zhang, "Multi-symplectic method for peakon-antipeakon collision of quasi-Degasperis-Procesi 
equation," Computer Physics Communications, vol. 185, no. 7, pp. 2020-2028, 2014.

[4] E. J. Parkes and B. R. Duffy, "An automated tanh-function method for finding solitary wave solutions to non-linear evolution equations," Computer Physics Communications, vol. 98, no. 3, pp. 288-300, 1996.

[5] C.-G. Zhu and W.-S. Kang, "Numerical solution of BurgersFisher equation by cubic B-Spline quasi-interpolation," Applied Mathematics and Computation, vol. 216, no. 9, pp. 2679-2686, 2010.

[6] A.-M. Wazwaz, "The tanh method for generalized forms of nonlinear heat conduction and Burgers-Fisher equations," Applied Mathematics and Computation, vol. 169, no. 1, pp. 321-338, 2005.

[7] A. N. Kolmogorov, I. G. Petrovskii, and N. S. Piskunov, "A study of the equation of diffusion with increase in the quantity of matter and its application to a biological problem," in Bull. Moscow Univ. Math. Mech., vol. 1, pp. 1-26, 6 edition, 1937.

[8] J. D. Murray, Mathematical Biology: I. An Introduction, vol. 2, Springer, NY, USA, 2002.

[9] J. Riordan, C. R. Doering, and D. Ben-Avraham, "Fluctuations and stability of sisher waves," Physical Review Letters, vol. 75, no. 3, pp. 565-568, 1995.

[10] S. Coen, M. Tlidi, P. Emplit, and M. Haelterman, "Convection versus dispersion in optical bistability," Physical Review Letters, vol. 83, no. 12, pp. 2328-2331, 1999.

[11] X. Y. Wang, Z. S. Zhu, and Y. K. Lu, "Solitary wave solutions of the generalised Burgers-Huxley equation," Journal of Physics A: Mathematical and General, vol. 23, no. 3, pp. 271-274, 1990.

[12] J. Satsuma, M. Ablowitz, B. Fuchssteiner, and M. Kruskal, “Topics in soliton theory and exactly solvable nonlinear equations," in Physical Review Letters, World Scientific, Singapore, Asia, 1987.

[13] A. C. Scott, Neurophysics, Wiley, NY, USA, 1977.

[14] X.-Y. Wang, "Nerve propagation and wall in liquid crystals," Physics Letters A, vol. 112, no. 8, pp. 402-406, 1985.

[15] A. L. Hodgkin and A. F. Huxley, "A quantitative description of membrane current and its application to conduction and excitation in nerve," The Journal of Physiology, vol. 117, no. 4, pp. 500-544, 1952.

[16] G. B. Whiteman, Linear and Nonlinear Waves, Wiley and Sons, NY, USA, 1974.

[17] M. Dehghan, J. M. Heris, and A. Saadatmandi, "Application of semi-analytic methods for the Fitzhugh-Nagumo equation, which models the transmission of nerve impulses," Mathematical Methods in the Applied Sciences, vol. 33, no. 11, pp. 1384-1398, 2010.

[18] J. Satsuma, "Exact solutions of Burgers' equation with reaction terms," in Topics in Soliton Theory And Exactly Solvable Nonlinear Equations, pp. 255-262, World Scientific Publishing, Singapore, Asia, 1987.

[19] W. Xinyi and L. Yuekai, "Exact solutions of the extended burgers-fisher equation," Chinese Physics Letters, vol. 7, no. 4, pp. 145-147, 1990.

[20] M. Javidi, "A numerical solution of the generalized BurgersHuxley equation by spectral collocation method," Applied Mathematics and Computation, vol. 178, no. 2, pp. 338-344, 2006.

[21] M. Javidi, "A numerical solution of the generalized Burger'sHuxley equation by pseudospectral method and Darvishi's preconditioning," Applied Mathematics and Computation, vol. 175, no. 2, pp. 1619-1628, 2006.
[22] M. Javidi and A. Golbabai, "A new domain decomposition algorithm for generalized Burger's-Huxley equation based on Chebyshev polynomials and preconditioning," Chaos, Solitons \& Fractals, vol. 39, no. 2, pp. 849-857, 2009.

[23] A. Golbabai and M. Javidi, "A spectral domain decomposition approach for the generalized Burger's-Fisher equation," Chaos, Solitons \& Fractals, vol. 39, no. 1, pp. 385-392, 2009.

[24] M. T. Darvishi, S. Kheybari, and F. Khani, "Spectral collocation method and Darvishi's preconditionings to solve the generalized Burgers-Huxley equation," Communications in Nonlinear Science and Numerical Simulation, vol. 13, no. 10, pp. 2091-2103, 2008.

[25] M. Sari, G. Gurarslan, and I. Dag, "A compact finite difference method for the solution of the generalized Burgers-Fisher equation," Numerical Methods for Partial Differential Equations, vol. 26, no. 1, pp. 125-134, 2010.

[26] D. A. Hammad and M. S. El-Azab, "2N order compact finite difference scheme with collocation method for solving the generalized Burgers-Huxley and Burgers-Fisher equations," Applied Mathematics and Computation, vol. 258, pp. 296-311, 2015.

[27] A. J. Khattak, "A computational meshless method for the generalized Burger's-Huxley equation," Applied Mathematical Modelling, vol. 33, no. 9, pp. 3718-3729, 2009.

[28] M. Sari and G. Gurarslan, "Numerical solutions of the generalized burgers-huxley equation by a differential quadrature method," Mathematical Problems in Engineering, vol. 2009, Article ID 370765, 11 pages, 2009.

[29] S. A. Malik, I. M. Qureshi, M. Amir, A. N. Malik, and I. Haq, "Numerical Solution to generalized Burgers'-Fisher equation using exp-function method hybridized with heuristic computation," PLoS ONE, vol. 10, no. 3, Article ID e0121728, 2015.

[30] M. Dehghan, B. N. Saray, and M. Lakestani, "Three methods based on the interpolation scaling functions and the mixed collocation finite difference schemes for the numerical solution of the nonlinear generalized Burgers-Huxley equation," Mathematical and Computer Modelling, vol. 50, no. 3-4, pp. 1129-1142, 2012.

[31] L. Zhang, L. Wang, and X. Ding, "Exact finite difference scheme and nonstandard finite difference scheme for Burgers and Burgers-Fisher equations," Journal of Applied Mathematics, vol. 2014, Article ID 597926, 12 pages, 2014.

[32] J. Biazar and F. Muhammadi, "Application of differential transform method to the generalized Burgers-Huxley equation," Applications and Applied Mathematics. An International Journal, vol. 5, no. 10, pp. 1726-1740, 2010.

[33] A. G. Bratsos, "A fourth order improved numerical scheme for the generalized Burgers-Huxley Equation," American Journal of Computational Mathematics, vol. 1, pp. 152-158, 2011.

[34] A. G. Bratsos, "An improved second-order numerical method for the generalized burgers-fisher equation," Anziam Journal, vol. 54, no. 3, pp. 181-199, 2013.

[35] M. Zarebnia and N. Aliniya, "A collocation method for numerical solution of the generalized Burgers-Huxley equation," Applied Mathematics and Computation, vol. 11, no. 8, pp. 687701, 2014.

[36] B. Batiha, M. S. M. Noorani, and I. Hashim, "Application of variational iteration method to the generalized Burger-Huxley equation," Chaos, Solitons \& Fractals, vol. 36, no. 3, pp. 660-663, 2008 . 
[37] O. O. Morufu, "An improved algorithm for the solution of generalized burger-Fisher equation," Applied Mathematics and Computation, vol. 5, pp. 1609-1614, 2014.

[38] I. Hashim, M. S. Noorani, and M. R. Said Al-Hadidi, "Solving the generalized Burgers-Huxley equation using the Adomian decomposition method," Mathematical and Computer Modelling, vol. 43, no. 11-12, pp. 1404-1411, 2006.

[39] T. Zhao, C. Li, Z. Zang, and Y. Wu, "Chebyshev-Legendre pseudo-spectral method for the generalised Burgers-Fisher equation," Applied Mathematical Modelling, vol. 36, no. 3, pp. 1046-1056, 2012.

[40] H. N. Ismail, K. Raslan, and A. A. Rabboh, "Adomian decomposition method for Burgers-Huxley and equation Burgers-Fisher equations," Applied Mathematics and Computation, vol. 159, no. 1, pp. 291-301, 2004.

[41] A. M. Al-Rozbayani and M. O. Al-Amr, "Discrete Adomian decomposition method for solving Burger's-Huxley equation," International Journal of Contemporary Mathematical Sciences, vol. 8, no. 13-16, pp. 623-631, 2013.

[42] B. Inan and A. R. Bahadir, "Numerical solutions of the generalized Burger-Huxley equation by implicit exponential finite difference method," Journal of Applied Mathematics, Statistics and Informatics, vol. 11, 2015.

[43] I. Celik, "Chebyshev Wavelet collocation method for solving generalized Burger-Huxley equation," Mathematical Methods in the Applied Sciences, vol. 39, no. 3, pp. 366-377, 2015.

[44] M. El-Kady, S. M. El-Sayed, and H. E. Fathy, "Development of Galerkin method for solving the generalized Burgers-Huxley equation," Mathematical Problems in Engineering, vol. 2013, Article ID 165492, 9 pages, 2013.

[45] B. Inan and A. R. Bahadir, "Numerical solution of the onedimensional Burgers' equation: Implicit and fully implicit exponential finite difference methods," Pramana-Journal of Physics, vol. 81, no. 4, pp. 547-556, 2013.

[46] I. Celik, "Haar wavelet method for solving generalized BurgersHuxley equation," Arab Journal of Mathematical Sciences, vol. 18, no. 1, pp. 25-37, 2012.

[47] J. E. Macias-Diaz, J. Ruiz-Ramirez, and J. Villa, “The numerical solution of a generalized burgers-Huxley equation through a conditionally bounder and symmetry-preserving method," Computers \& Mathematics with Applications. An International Journal, vol. 61, no. 11, pp. 3330-3342, 2011.

[48] S. Zhou and X. Cheng, "A linearly semi-implicit compact scheme for the Burgers-Huxley equation," International Journal of Computer Mathematics, vol. 88, no. 4, pp. 795-804, 2011.

[49] V. J. Ervin, J. E. Macias-Diaz, and J. Ruiz-Ramirez, "A positive and bounded finite element approximation of the generalized Burgers-Huxley equation," Journal of Mathematical Analysis and Applications, vol. 424, no. 2, pp. 1143-1160, 2015.

[50] G.-W. Wang, X.-Q. Liu, and Y.-Y. Zhang, "New explicit solutions of the generalized Burgers-Huxley equation," Vietnam Journal of Mathematics, vol. 41, no. 2, pp. 161-166, 2013.

[51] K. M. Owolabi, "Numerical solution of the generalized BurgersHuxley equation by exponential time differencing scheme," International Journal of Biomedical Engineering and Science, vol. 1, pp. 43-52, 2015.

[52] M. Sari, G. Gurarslan, and A. Zeytinoglu, "High-order finite difference schemes for numerical solutions of the generalized Burgers-Huxley equation," Numerical Methods for Partial Differential Equations, vol. 27, no. 5, pp. 1313-1326, 2011.
[53] R. C. Mittal and A. Tripathi, "Numerical solutions of generalized Burgers-Fisher and generalized Burgers-Huxley equations using collocation of cubic B-Splines,' International Journal of Computer Mathematics, vol. 92, no. 5, pp. 1053-1077, 2015.

[54] R. C. Mittal and R. K. Jain, "Numerical solutions of nonlinear Burgers' equation with modified cubic B-Splines collocation method," Applied Mathematics and Computation, vol. 218, no. 15, pp. 7839-7855, 2012.

[55] B. K. Singh, G. Arora, and M. K. Singh, "A numerical scheme for the generalized Burgers-Huxley equation," Journal of the Egyptian Mathematical Society, vol. 24, no. 4, pp. 629-637, 2016.

[56] M. Reza, "B-spline collocation algorithm for numerical solution of the generalized Bueger's-Huxley equation," Numerical Methods for Partial Differential Equations, vol. 29, no. 4, pp. 1173-1191, 2013.

[57] M. Reza, "Spline solution of the generalized Burgers-Fisher equation," Applicable Analysis, vol. 91, no. 12, pp. 2189-2215, 2012.

[58] M. Bukhari, M. Arshad, S. Batool, and S. M. Saqlain, "Numerical solution of generalized Burger's-Huxley equation using local radial basis functions," International Journal of Advanced and Applied Sciences, vol. 4, no. 5, pp. 1-11, 2017.

[59] M. Yaseen, M. Abbas, A. I. Ismail, and T. Nazir, "A cubic trigonometric B-spline collocation approach for the fractional subdiffusion equations," Applied Mathematics and Computation, vol. 293, pp. 311-319, 2017.

[60] T. Nazir, M. Abbas, A. I. M. Ismail, A. A. Majid, and A. Rashid, "The numerical solution of advection-diffusion problems using new cubic trigonometric B-splines approach," Applied Mathematical Modelling, vol. 40, no. 7-8, pp. 4586-4611, 2016.

[61] M. Abbas, A. A. Majid, A. I. Md Ismail, and A. Rashid, "Numerical method using cubic B-spline for a strongly coupled reaction-diffusion system," PLoS ONE, vol. 9, no. 1, Article ID e83265, 2014.

[62] M. Abbas, A. A. Majid, A. I. Ismail, and A. Rashid, "The application of cubic trigonometric B-spline to the numerical solution of the hyperbolic problems," Applied Mathematics and Computation, vol. 239, pp. 74-88, 2014.

[63] M. Abbas, A. A. Majid, A. I. M. Ismail, and A. Rashid, "Numerical method using cubic trigonometric B-Spline technique for non-classical diffusion problem," Abstract and Applied Analysis, vol. 2014, Article ID 849682, 10 pages, 2014.

[64] S. Mat Zin, A. Abd Majid, A. I. M. Ismail, and M. Abbas, "Application of hybrid cubic B-spline collocation approach for solving a generalized nonlinear Klien-Gordon equation," Mathematical Problems in Engineering, vol. 2014, Article ID 108560, 10 pages, 2014.

[65] P. M. Prenter, Splines and Variational Methods, Wiley Classics Library, John Wiley and Sons, NY, USA, 1989.

[66] C. De Boor, A Practical Guide to Splines (Applied Mathematical Sciences), Springer, NY, USA, 1978.

[67] D. U. V. Rosenberg, Methods for solution of partial differential equations, American Elsevier Publishing, NY, USA, 1969.

[68] S. S. Siddiqi and S. Arshed, "Quintic B-spline for the numerical solution of the good Boussinesq equation," Journal of the Egyptian Mathematical Society, vol. 22, no. 2, pp. 209-213, 2014.

[69] R. Nawaz, H. Ullah, S. Islam, and M. Idrees, "Application of optimal homotopy asymptotic method to burger equations," Journal of Applied Mathematics, vol. 2013, Article ID 387478, 8 pages, 2013. 
[70] J. He, "Variational iteration method-A kind of non-linear analytical technique: some examples," International Journal of Non-Linear Mechanics, vol. 34, no. 4, pp. 699-708, 1999. 


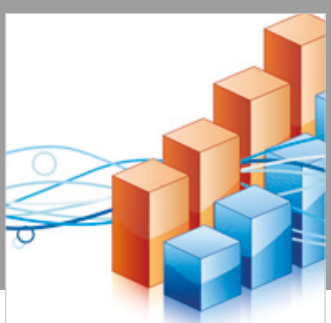

Advances in

Operations Research

\section{-n-m}
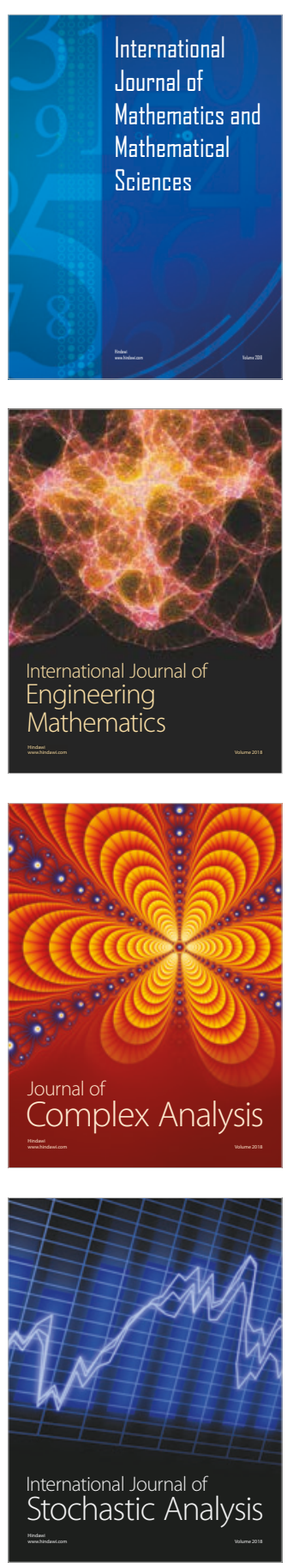
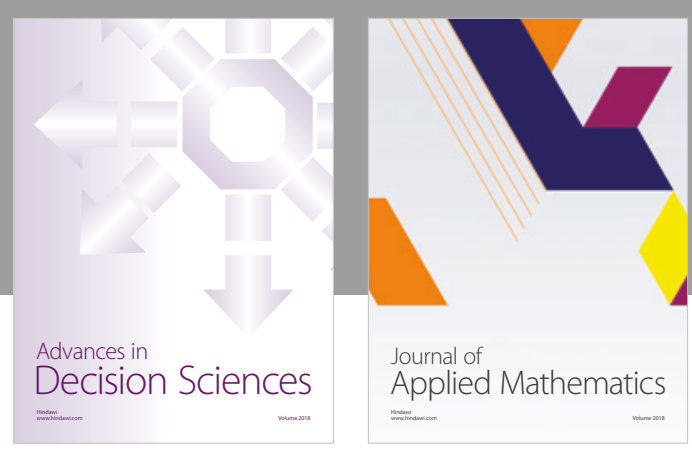

Journal of

Applied Mathematics
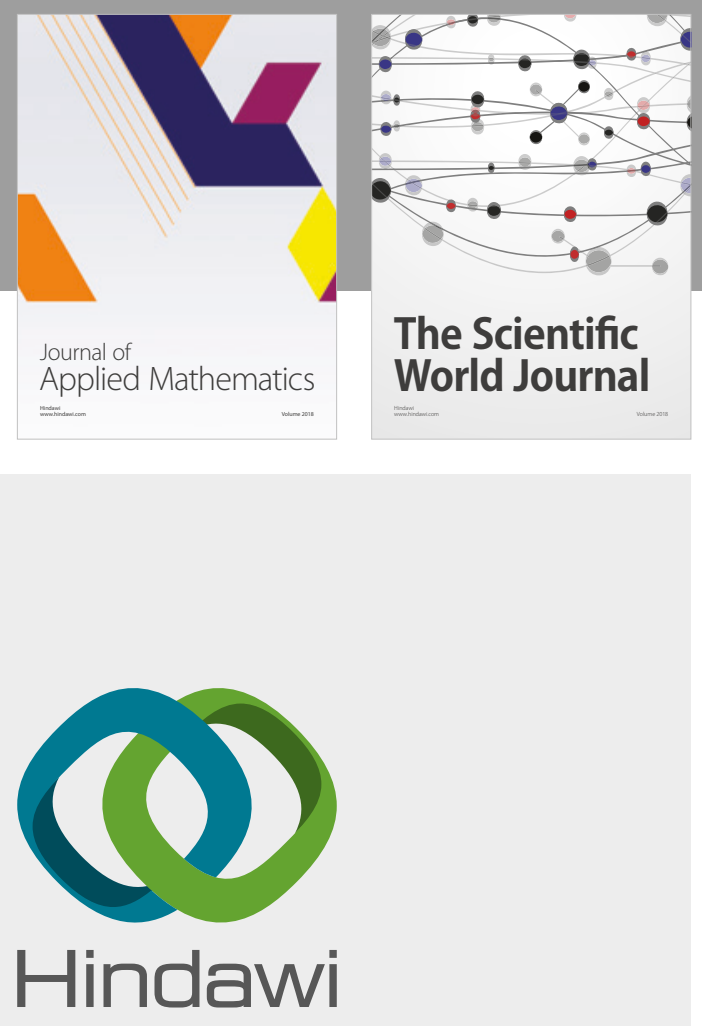

Submit your manuscripts at

www.hindawi.com

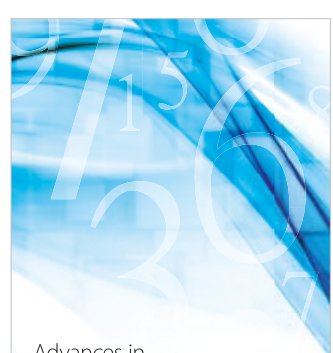

Advances in
Numerical Analysis
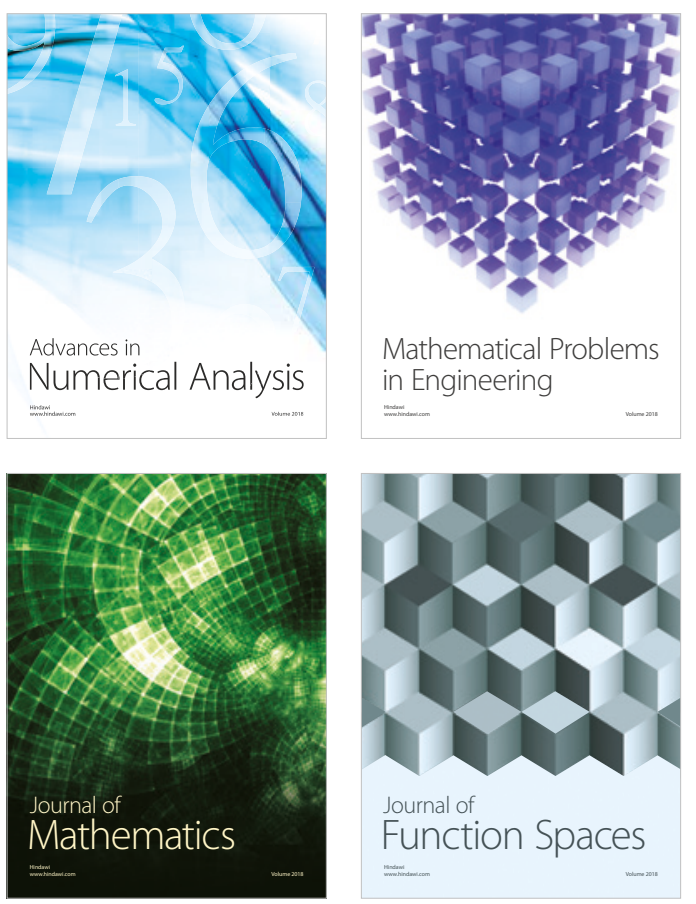

Mathematical Problems in Engineering

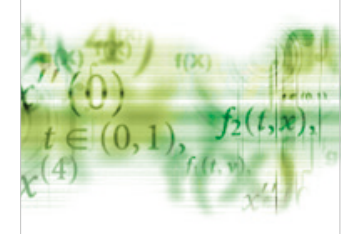

International Journal of

Differential Equations

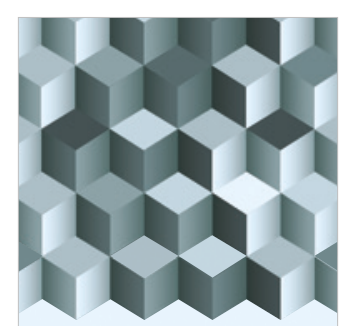

Journal of

Function Spaces

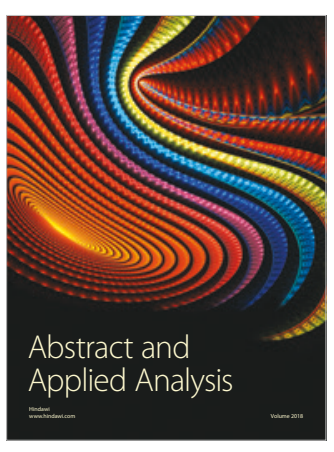

The Scientific

World Journal

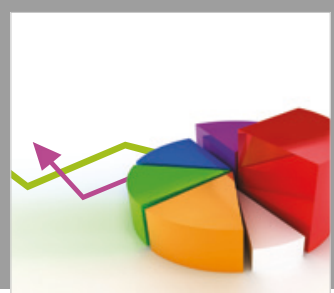

Journal of

Probability and Statistics
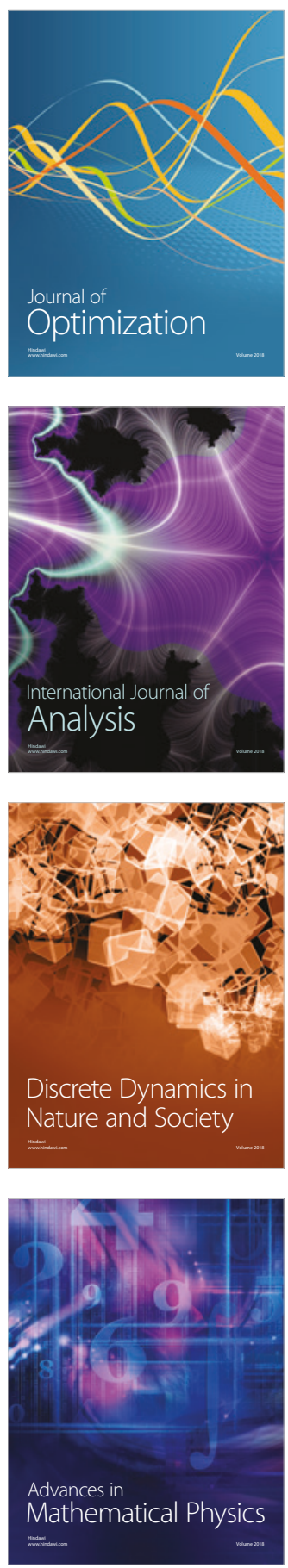